\title{
Data report: paleomagnetic measurements of igneous rocks from Shatsky Rise Expedition 324'
}

\author{
Margaret Pueringer, ${ }^{2}$ William Sager, ${ }^{2,3}$ and Bernard Housen ${ }^{4}$
}

\section{Chapter contents}

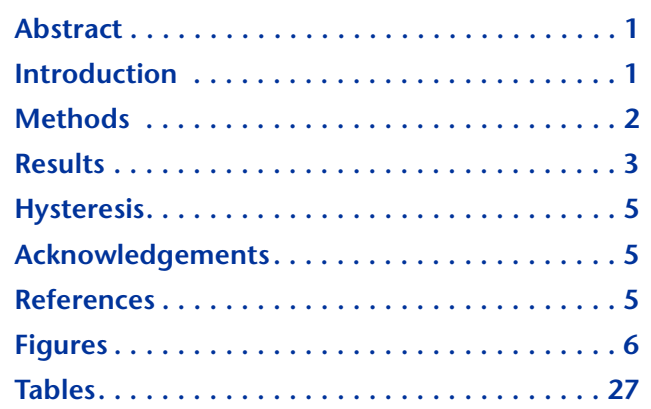

1 Pueringer, M., Sager, W., and Housen, B., 2013. Data report: paleomagnetic measurements of igneous rocks from Shatsky Rise Expedition 324. In Sager, W.W., Sano, T., Geldmacher, J., and the Expedition 324 Scientists, Proc. IODP, 324: Tokyo (Integrated Ocean Drilling Program Management International, Inc.).

doi:10.2204/iodp.proc.324.202.2013

2Department of Geology and Geophysics, Texas A\&M University, College Station TX 77843, USA. ${ }^{3}$ Current address: Department of Earth and Atmospheric Sciences, University of Houston, Houston TX 77204, USA. wwsager@uh.edu ${ }^{4}$ Department of Geology, Western Washington University, Bellingham WA 98225, USA.

\section{Abstract}

Five sites were cored on Shatsky Rise, an oceanic plateau, during Integrated Ocean Drilling Program Expedition 324 to gain a better understanding of its formation processes. To augment and improve the shipboard paleomagnetic results, an additional 135 samples from Sites U1346-U1348 and U1350 were measured at the Northwest Paleomagnetism Laboratory at Western Washington University (USA). Samples from all sites were demagnetized using either alternating field (AF) or thermal demagnetization in approximately equal numbers. Most samples display a downwarddirected overprint likely caused by the drill string magnetic field. This overprint was typically removed by 10-15 mT AF demagnetization or $\sim 300^{\circ} \mathrm{C}$ thermal demagnetization. AF demagnetization generally worked well to isolate a characteristic remanence direction, with samples mostly showing low $(<10 \mathrm{mT})$ to moderate (10-20 mT) median destructive field values. Thermal demagnetization was also mostly successful in isolating a characteristic remanence at temperatures $>350^{\circ}-400^{\circ} \mathrm{C}$. Some samples showed small sections of self-reversal during thermal demagnetization. Changes in inclination over depth showed little variation at Sites U1346 and U1347, but more variation was recorded at Site U1350. Hysteresis analysis showed that samples from all sites reside in the single-domain to pseudosingle domain region of the Day plot.

\section{Introduction}

Shatsky Rise is an oceanic plateau that is a large igneous province (LIP) on the Pacific plate thought to be the product of a mantlemelting anomaly interacting with a triple junction. It was formed in Late Jurassic to Early Cretaceous time (Sager et al., 1999; Nakanishi et al., 1999). Three volcanic massifs, Tamu, Ori, and Shirshov, and the Papanin Ridge are the major structures of Shatsky Rise (Fig. F1). Based on magnetic lineations surrounding Shatsky Rise, the age and volume of the plateau decreases from the Tamu Massif to the Papanin Ridge (Sager et al., 1999).

Because Shatsky Rise volcanism was poorly understood, coring on Shatsky Rise during Expedition 324 gathered samples of igneous rocks to better understand its formation. Basaltic lava flows were recovered at four sites (U1346, U1347, U1349, and U1350), whereas volcaniclastic material was recovered at a fifth site (U1348) (Fig. F1) (Sager et al., 2011a, 2011b; see also "Expedition 324 summary" chapter [Expedition 324 Scientists, 2010]). Prior 
to Expedition 324, significant basaltic material had been cored only once, at Ocean Drilling Program Site 1213 on the south flank of Tamu Massif. At this site, basalt from three massive flows was recovered over a $47 \mathrm{~m}$ section (Fig. F2) (Koppers et al., 2010). At Site U1347, also on Tamu Massif, a basement section $159.9 \mathrm{~m}$ in length produced both pillow and massive flows, but at Site U1348, on the north flank of Tamu Massif, only volcaniclastics were recovered from $\sim 120 \mathrm{~m}$ of core. Two holes at Ori Massif both yielded basalt flows: thin massive flows from $85.3 \mathrm{~m}$ of core at Site U1349 and $172.7 \mathrm{~m}$ of pillow and thin massive flows from Site U1350 (Fig. F2). Site U1346, on Shirshov Massif, yielded a $52.6 \mathrm{~m}$ section of pillow lavas with two small massive flows.

One of the goals of Expedition 324 was to understand the volcanism that built Shatsky Rise. Paleomagnetic measurements can aid in this understanding because the measured paleomagnetic inclinations give clues about the timing and paleolatitude of eruptions. In this study, the remanent magnetization of igneous rock samples was measured to determine changes in inclination with depth at each site. Changes in remanent inclination versus depth from an igneous section constrain the eruptive time span by the amount of observed geomagnetic field secular variation. The main focus of this report is measurements from basalt lava flows from Sites U1346, U1347, and U1350. A small number of samples from Site U1348 were measured to see if the hyaloclastite samples from that hole would produce reliable results (they did not). We did not measure samples from Site U1349 because another member of the shipboard party is working on that site. Paleomagnetic analysis for 120 basalt samples was done onboard the $\mathrm{R} / \mathrm{V}$ JOIDES Resolution during Expedition 324. In our data tables, we combine those results with ours for completeness.

\section{Methods}

A total of 135 samples were measured in the Pacific Northwest Paleomagnetism Laboratory, which contains a Lodestar Magnetics Shielded Room, at Western Washington University (WWU) (USA). All samples were $2 \mathrm{~cm} \times 2 \mathrm{~cm} \times 2 \mathrm{~cm}$ cubes cut from the working half of Expedition 324 basalt cores. Magnetic susceptibility was measured on all samples prior to demagnetization, using an AGICO KLY3-S magnetic susceptibility bridge. Subsequently, the natural remanent magnetization (NRM) was measured in an AGICO JR6 dual-speed spinner magnetometer. Because the demagnetization results from some samples of massive flows were erratic in shipboard measurements, samples from massive units were dunked in liquid nitrogen for 20 min 1-4 times to remove overprint magnetizations resulting from multidomain magnetite and hematite. All samples were demagnetized using either alternating field (AF) demagnetization with a DTech D2000 AF demagnetizer or thermal demagnetization in an ASC Model TD48 or ASC Model TD48-SC thermal demagnetizing oven. A vibrating sample magnetometer (VSM; Princeton Measurements Corporation MicroMag Model 3900) was used to produce hysteresis loops on four samples from each site that were judged representative of the site's flow units.

Pilot batches of samples using AF and thermal demagnetization from all sites showed little difference in the efficacy of removing overprint magnetizations, so approximately half of the samples from each site were demagnetized thermally (62 samples) and the other half using AF demagnetization (73 samples). Initially, thermal pilot samples were separated into two batches of 12 samples, one set being demagnetized in an argon atmosphere and the other in a natural atmosphere to compare the effects of oxidation. Because there was no difference in the results from the two treatments, the remaining samples were demagnetized in a natural atmosphere. AF demagnetization started at $5 \mathrm{mT}$, with steps of $5 \mathrm{mT}$ up to 60-120 mT. Thermal demagnetization started at $80^{\circ} \mathrm{C}$, with steps of $40^{\circ} \mathrm{C}$ up to $500^{\circ} \mathrm{C}$ and steps of $20^{\circ}-25^{\circ} \mathrm{C}$ above $500^{\circ} \mathrm{C}$. Complications arose with the JR6 magnetometer in that some of the thermally demagnetized samples had magnetizations too low to measure with this magnetometer above a certain step. If it was not possible to demagnetize a sample past $70 \%$ of the original magnetic intensity, the result was not considered a valid measurement of the characteristic remanence.

After samples were demagnetized, the data were analyzed using Remasoft 3.0 software. Equal angle spherical projections, demagnetization intensity plots, and Zijderveld plots (see Tauxe, 1998) were made for each sample. Principal component analysis (PCA) directions (Kirschvink, 1980) were calculated from demagnetization data, typically using higher level demagnetization steps that trend toward the $\mathrm{Zij}$ derveld plot origin. This result is considered the characteristic or primary magnetization for the sample. PCA solutions were calculated both anchored to the origin and not anchored (Tables T1, T2, T3). VSM hysteresis loops were analyzed with the Micromag AGM-VSM program. A Day plot (Day et al., 1977) was produced using the hysteresis values saturation remanent magnetization $\left(M_{\mathrm{rs}}\right)$ /saturation magnetization $\left(M_{\mathrm{s}}\right)$ vs. remanent coercivity $\left(H_{\mathrm{cr}}\right) /$ coercivity $\left(H_{\mathrm{c}}\right)$.

Measurements done during Expedition 324 included $\mathrm{AF}$ and thermal demagnetization and bulk magnetic 
susceptibility measurements recorded during the thermal demagnetization process. Although paleomagnetic work was done on board the ship for all sites, in this report we concentrate only on the data from Sites U1346-U1348 and U1350.

\section{Results \\ Site U1346}

A total of 31 samples were measured from Site U1346 cores, but only 29 samples produced good results (Table T1). All samples are aphyric basalts from pillow flows. Both AF and thermal demagnetization techniques were used to isolate the primary magnetization. WWU measurements were divided into seven samples measured with $\mathrm{AF}$ demagnetization and eight examined with thermal demagnetization. Most samples have a drilling overprint that is removed at $\sim 5-10 \mathrm{mT}$ during AF demagnetization and at $\sim 200^{\circ}-300^{\circ} \mathrm{C}$ during thermal demagnetization. The AF demagnetized rocks show two responses, one type with low median destructive field (MDF; $<10 \mathrm{mT})$ and the other with a higher MDF ( $>10 \mathrm{mT})$ (Figs. F3, F4). After the drilling overprint is removed, the samples usually display univectorial decay to the origin, with a few samples showing erratic behavior at high demagnetizing steps. The samples that show a MDF of $<10 \mathrm{mT}$ are $>90 \%$ demagnetized by 20 $30 \mathrm{mT}$ (Fig. F4), whereas the samples with a MDF of $>10 \mathrm{mT}$ are $>90 \%$ demagnetized by $40-50 \mathrm{mT}$ (Fig. F3). Some thermally demagnetized samples show a small range of temperatures with partial self-reversal at $\sim 300^{\circ} \mathrm{C}$ (e.g., Doubrovine and Tarduno, 2004, 2005) and/or a large overprint that is removed at $\sim 200^{\circ} \mathrm{C}$ (Fig. F5). After removal of the overprint or at temperatures above the self-reversal portion of demagnetization, most of the samples display univectorial decay to the origin. All thermally demagnetized samples were $>90 \%$ demagnetized at $450^{\circ}-500^{\circ} \mathrm{C}$. Of the 15 samples measured at WWU, 13 samples produced good results and were judged as samples giving a consistent principal direction that points toward the origin with a maximum angular deviation (MAD) of $<10^{\circ}$. The other 16 samples measured during Expedition 324 showed similar behaviors in both $\mathrm{AF}$ and thermal demagnetizations (see the "Expedition 324 summary" chapter [Expedition 324 Scientists, 2010]). Four samples were dunked in liquid nitrogen as a test to determine its effect on sample overprints. Very little change was apparent in the magnetic intensity of the rocks, at most $0.5 \mathrm{~A} / \mathrm{m}$ (Table T4). There were no noticeable effects of the low-temperature treatment on sample behavior during demagnetization.

Sample inclinations calculated using anchored and unanchored PCA solutions showed negligibly differ- ent values. The inclinations vary little throughout the length of the cored section, except for one outlier sample with an inclination of $27^{\circ}$ (Fig. F6). Ignoring the single outlier, the average inclination is $-21^{\circ}$, with a standard deviation of $5.7^{\circ}$. The lack of downhole variation and the low standard deviation imply that the entire section is recording essentially the same magnetic direction, so very little paleosecular variation was recorded, and the section was likely erupted in a short period of time.

\section{Site U1347}

From Site U1347 cores, 126 samples were measured, 61 during Expedition 324 and 65 at WWU. Only 119 samples produced good results (Table T2). The samples are aphyric and plagioclase phyric basalts from massive flows and pillow flow units. To isolate primary magnetization, both AF and thermal demagnetization techniques were applied to different subsets of samples. At WWU, 32 of the 65 samples were demagnetized using AF demagnetization and 33 were demagnetized using thermal demagnetization. The demagnetized samples have varying amounts of drilling overprint. In the AF demagnetized samples, the drilling overprint is typically directed vertically downward (a sign of drill pipe remagnetization; e.g., Fuller et al., 2006) and is removed after 10-15 mT. For thermally demagnetized samples, the overprint is usually removed progressively up to $310^{\circ}-360^{\circ} \mathrm{C}$. For both demagnetization types, the overprint was apparently never removed entirely from a small number of samples. Two responses observed with $\mathrm{AF}$ demagnetization are samples with low MDF and samples with higher MDF (Figs. F7, F8). The lowMDF samples are $>90 \%$ demagnetized at $30 \mathrm{mT}$ and the higher MDF samples are $>90 \%$ demagnetized at 40-60 mT. Once the overprint is removed, most samples display univectoral decay toward the Zijderveld plot origin. In the lower cores (324-U1347A-25R through 29R), which sampled thick massive flows, sample directions become erratic at AF steps above $50 \mathrm{mT}$.

The thermally demagnetized samples displayed two different demagnetization behaviors. For one group, the intensity versus temperature curve shows a sharp decline around $300^{\circ} \mathrm{C}$. In the other group, a nearly linear decrease in magnetic intensity is seen throughout the measurements (Figs. F9, F10). Some samples showed erratic directions in steps above $520^{\circ} \mathrm{C}$, especially samples from Cores 324-U1347A$25 \mathrm{R}$ through $29 \mathrm{R}$. For a few samples, small partial self-reversal sections occurred in demagnetization steps around $300^{\circ} \mathrm{C}$ (e.g., Doubrovine and Tarduno 2004, 2005) (Fig. F11). Typically, the sample increased in intensity for only 1-3 demagnetization 
steps, and the intensity increase was only $10 \%-15 \%$ of the NRM.

Of the samples measured at WWU, 59 of the 65 produced good PCA solutions. Once again, there was little difference between solutions calculated with and without being anchored to the origin. The good results have a MAD of $<10^{\circ}$ and display a consistent direction after overprint removal. Shipboard AF demagnetization results were similar, but the thermally demagnetized samples displayed much more erratic behavior. Of the shipboard measurements, characteristic remanence directions for 48 of the 60 with MAD $<10^{\circ}$ were judged to have produced reliable results (Table T2).

Low-temperature treatment was applied to 39 samples at WWU (Table T5). The massive flow units had the greatest change in magnetic intensity. Samples that showed a large drop of intensity were dunked more than once to assure full removal of multidomain magnetite and hematite overprints. Because this treatment was used to reduce the effect of overprint acquired by multidomain magnetic grains, this result implies that multidomain grains contribute significantly to the magnetization of these units. The thin inflation units displayed little change in intensity with low-temperature dunking, implying that they have few multidomain grains contributing to their magnetization.

Site U1347 characteristic remanence inclinations are mostly low and positive (Fig. F12). Samples from the uppermost $\sim 15 \mathrm{~m}$ of the basement ( 160-175 mbsf) and lowermost $\sim 40 \mathrm{~m}$, below $\sim 270 \mathrm{mbsf}$, show higher scatter than elsewhere, mostly because of greater scatter in thermal demagnetization results. Samples appear to show at least three groups of inclinations. Between $\sim 175$ and $210 \mathrm{mbsf}$, the average inclination is $20^{\circ}-30^{\circ}$, and the same is true for the section between $\sim 240$ and 270 mbsf. In between, the average inclination appears shallower, $\sim 10^{\circ}-15^{\circ}$. Sample inclination scatter is high in the uppermost $\sim 15 \mathrm{~m}$ and lowermost $\sim 40 \mathrm{~m}$, so we cannot tell whether those samples give a significantly different inclination than the middle section without further analysis.

\section{Site U1348}

Only five volcaniclastic samples from Site U1348 were measured as a test. Only three samples were strong enough to measure NRM. Of the three samples, two were measured using thermal demagnetization and one was measured using AF demagnetization. Demagnetization did not produce consistent magnetization directions, so the Site U1348 section was considered unsuitable for further study.

\section{Site U1350}

A total of 109 samples were measured from Site U1350 cores, 42 samples measured during Expedition 324 and 67 measured at WWU. Good results were produced from 102 samples (Table T3). At WWU, 33 samples were demagnetized using AF demagnetization and 34 using thermal demagnetization. The AF demagnetized samples showed two responses similar to the previous sites, low MDF (Fig. F13) and higher MDF (Fig. F14). Both types of samples display univectorial decay after the overprint is removed. The amount of overprint correlates with the MDF. A low-MDF sample typically displays a large overprint, whereas a higher MDF sample displays smaller overprint. The thermally demagnetized samples displayed two behaviors, one with a small partial self-reversal at $300^{\circ} \mathrm{C}$ (e.g., Doubrovine and Tarduno, 2004, 2005) and one without (Figs. F15, F16). Some thermally demagnetized samples showed erratic behavior above $500^{\circ} \mathrm{C}$. Varying degrees of overprint were evident, but for most samples the overprint did not have a significant effect on the measured direction. After the overprint was removed, samples with both thermal demagnetization behaviors showed univectorial decay to the origin. Of the 67 samples, 63 yielded a consistent primary magnetization with a MAD $<10^{\circ}$. As with samples from other sites, no significant difference existed between PCA solutions using the origin as an anchor and those without an anchor. The 42 samples that were measured during Expedition 324 gave similar AF results, but the thermally demagnetized samples measured on the ship show a much more erratic behavior than those measured at WWU. Results from samples with consistent directional behavior were similar to those measured at WWU. A total of 39 of the 42 samples had a MAD $<10^{\circ}$ and are considered to have produced reliable results (Table T3). In all, 32 samples were treated by dunking in liquid nitrogen to remove the effects of multidomain grains, but the effects were negligible (Table T6).

A plot of inclination versus depth shows that inclinations are all close to zero with slightly negative inclinations being the norm (Fig. F17). AF and thermal demagnetized sample inclinations give similar values, but results from thermal demagnetization are more erratic between $\sim 195$ and 235 mbsf. Inclinations at the top and bottom of the hole appear indistinguishable, but the section between 195 and 235 mbsf may have a more positive average inclination. Because this is also the section with higher scatter, this inference cannot be confirmed without further analysis; it may be that all samples from Site U1350 record the same inclination. 


\section{Hysteresis}

Hysteresis loops of four samples each from Sites U1346, U1347, and U1350 were made with the VSM (Figs. F18, F19, F20). All hysteresis loops show rapid saturation of the magnetization with increasing field strength and only a small amount of hysteresis. This type of response is typical of titanomagnetite grains. On a Day plot (Day et al., 1977) $M_{\mathrm{rs}} / M_{\mathrm{s}}$ versus $H_{\mathrm{cr}} / H_{\mathrm{c}}$ values for most samples plot in the field that defines pseudosingle-domain behavior (Table T7; Fig. F21). This observation implies that the grains in the samples are either pseudosingle-domain-sized grains or a mixture of multidomain and single-domain grains. Samples from Sites U1346 and U1347 display a distribution that is nearly linear from upper left (lower $H_{\mathrm{cr}} / H_{\mathrm{c}}$ and higher $M_{\mathrm{rs}} / M_{\mathrm{s}}$ ) to lower right (higher $H_{\mathrm{cr}} /$ $H_{\mathrm{c}}$ and lower $\left.M_{\mathrm{rs}} / M_{\mathrm{s}}\right)$, similar to mixtures of singleand multidomain grains, suggesting that these samples have a simple distribution of similar magnetic grains of different sizes (Dunlop, 2002a, 2002b). The distribution of the Site U1350 samples is not linear, suggesting a more complex set of magnetic grains. In addition, samples from Site U1347 plot farthest toward the upper left, whereas samples from Site U1346 plot toward the bottom right. This observation implies that single- and pseudosingle-domain behavior is strongest for Sites U1347 and U1350, whereas the samples from Site U1346 display more influence from multidomain grains.

\section{Acknowledgments}

This research used samples and data provided by the Integrated Ocean Drilling Program (IODP).

\section{References}

Day, R., Fuller, M., and Schmidt, V.A., 1977. Hysteresis properties of titanomagnetites: grain-size and compositional dependence. Phys. Earth Planet. Inter., 13(4):260267. doi:10.1016/0031-9201(77)90108-X

Doubrovine, P.V., and Tarduno, J.A., 2004. Self-reversed magnetization carried by titanomaghemite in oceanic basalts. Earth Planet. Sci. Lett., 222(3-4):959-969. doi:10.1016/j.epsl.2004.04.009

Doubrovine, P.V., and Tarduno, J.A., 2005. On the compositional field of self-reversing titanomaghemite: constraints from Deep Sea Drilling Project Site 307. J. Geophys. Res.: Solid Earth, 110(B11):B11104. doi:10.1029/2005JB003865

Dunlop, D.J., 2002a. Theory and application of the Day plot $\left(M_{\mathrm{rs}} / M_{\mathrm{s}}\right.$ versus $\left.H_{\mathrm{cr}} / H_{\mathrm{c}}\right), 1$. Theoretical curves and tests using titanomagnetite data. J. Geophys. Res.: Solid Earth, 107(B3):2056. doi:10.1029/2001JB000486

Dunlop, D.J., 2002b. Theory and application of the Day plot $\left(M_{\mathrm{rs}} / M_{\mathrm{s}}\right.$ versus $\left.H_{\mathrm{cr}} / H_{\mathrm{c}}\right), 2$. Application to data for rocks, sediments, and soils. J. Geophys. Res.: Solid Earth, 107(B3):2057. doi:10.1029/2001JB000487

Expedition 324 Scientists, 2010. Expedition 324 summary. In Sager, W.W., Sano, T., Geldmacher, J., and the Expedition 324 Scientists, Proc. IODP, 324: Tokyo (Integrated Ocean Drilling Program Management International, Inc.). doi:10.2204/iodp.proc.324.101.2010

Fuller, M., Molina-Garza, R., Touchard, Y., and Kidane, T., 2006. Paleomagnetic records from carbonate legs in the Southern Oceans and attendant drilling and coring related effects. In Sager, W.W., Acton, G.D., Clement, B.M., and Fuller, M. (Eds.), ODP Contributions to Paleomagnetism. Phys. Earth Planet. Int., 156(3-4):242-260. doi:10.1016/j.pepi.2005.08.007

Kirschvink, J.L., 1980. The least-squares line and plane and the analysis of palaeomagnetic data. Geophys. J. $R$. Astron. Soc., 62(3):699-718. doi:10.1111/j.1365246X.1980.tb02601.x

Koppers, A.A.P., Sano, T., Natland, J.H., Widdowson, M., Almeev, R., Greene, A.R., Murphy, D.T., Delacour, A., Miyoshi, M., Shimizu, K., Li, S., Hirano, N., Geldmacher, J., and the Expedition 324 Scientists, 2010. Massive basalt flows on the southern flank of Tamu Massif, Shatsky Rise: a reappraisal of ODP Site 1213 basement units. In Sager, W.W., Sano, T., Geldmacher, J., and the Expedition 324 Scientists, Proc. IODP, 324: Tokyo (Integrated Ocean Drilling Program Management International, Inc.). doi:10.2204/iodp.proc.324.109.2010

Nakanishi, M., Sager, W.W., and Klaus, A., 1999. Magnetic lineations within Shatsky Rise, northwest Pacific Ocean: implications for hot spot-triple junction interaction and oceanic plateau formation. J. Geophys. Res.: Solid Earth, 104(B4):7539-7556. doi:10.1029/1999JB900002

Sager, W.W., Kim, J., Klaus, A., Nakanishi, M., and Khankishieva, L.M., 1999. Bathymetry of Shatsky Rise, northwest Pacific Ocean: implications for ocean plateau development at a triple junction. J. Geophys. Res.: Solid Earth, 104(4):7557-7576. doi:10.1029/1998JB900009

Sager, W.W., Sano, T., and Geldmacher, J., 2011a. How do oceanic plateaus form? Clues from drilling at Shatsky Rise. Eos, Trans. Am. Geophys. Union, 92(5):37-38. doi:10.1029/2011E0050001

Sager, W.W., Sano, T., Geldmacher, J., and the IODP Expedition 324 Scientists, 2011b. IODP Expedition 324: ocean drilling at Shatsky Rise gives clues about oceanic plateau formation. Sci. Drill., 12:24-31. doi:10.2204/ iodp.sd.12.03.2011

Tauxe, L., 1998. Paleomagnetic Principles and Practice: Dordrecht, Netherlands (Kluwer Academic Publishers).

Initial receipt: 1 August 2012

Acceptance: 22 April 2013

Publication: 19 August 2013

MS 324-202 
Figure F1. Map of Shatsky Rise showing the location of IODP Expedition 324 Sites U1346-U1350 and ODP Site 1213. Depths above $5 \mathrm{~km}$ are shaded. Gray lines show magnetic lineations and fracture zones (Nakanishi et al., 1999).

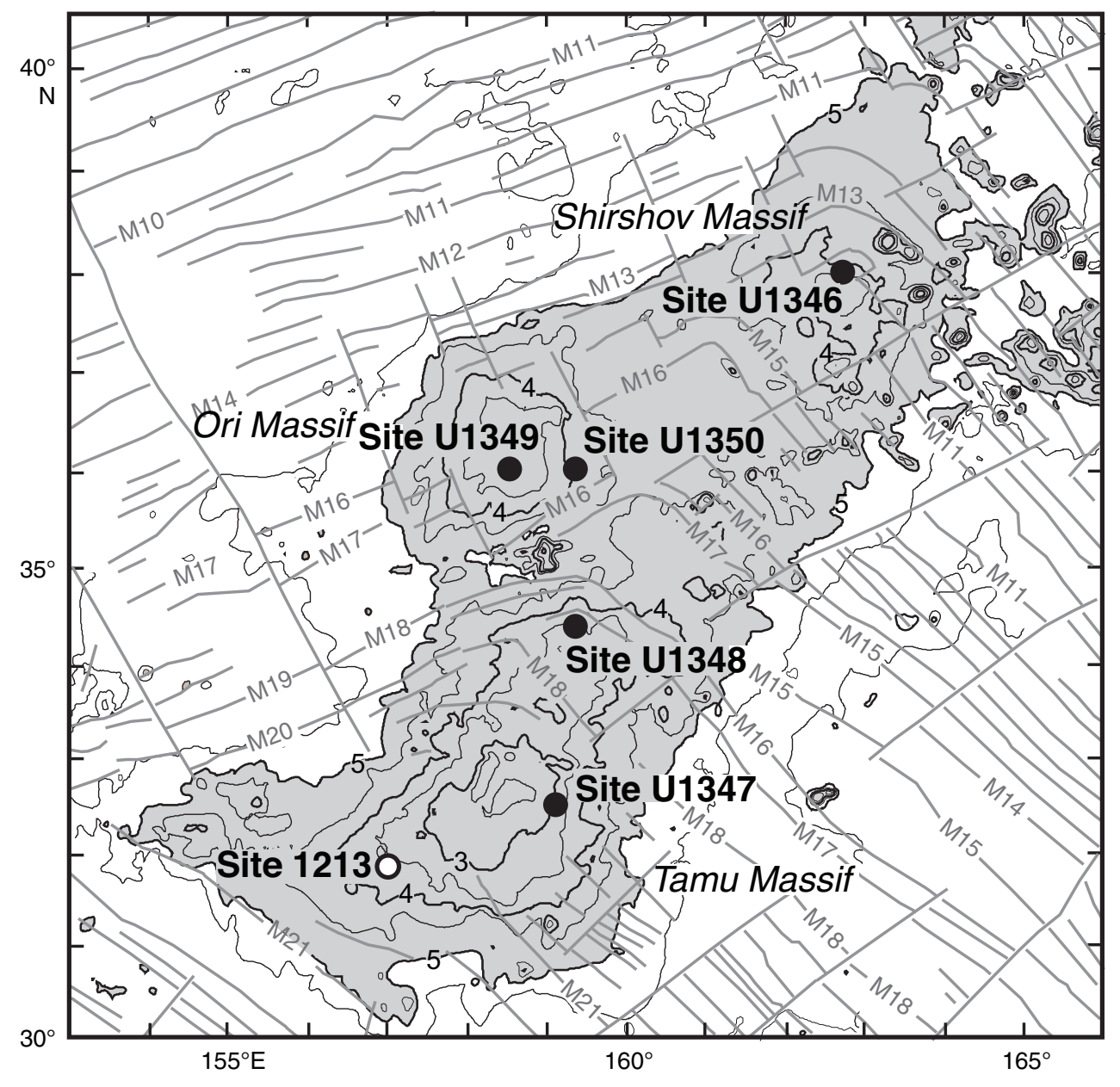


Figure F2. Graphic lithology sections for IODP Expedition 324 sites and ODP Site 1213. Roman numerals indicate lithologic units (see the "Expedition 324 summary" chapter [Expedition 324 Scientists, 2010]).

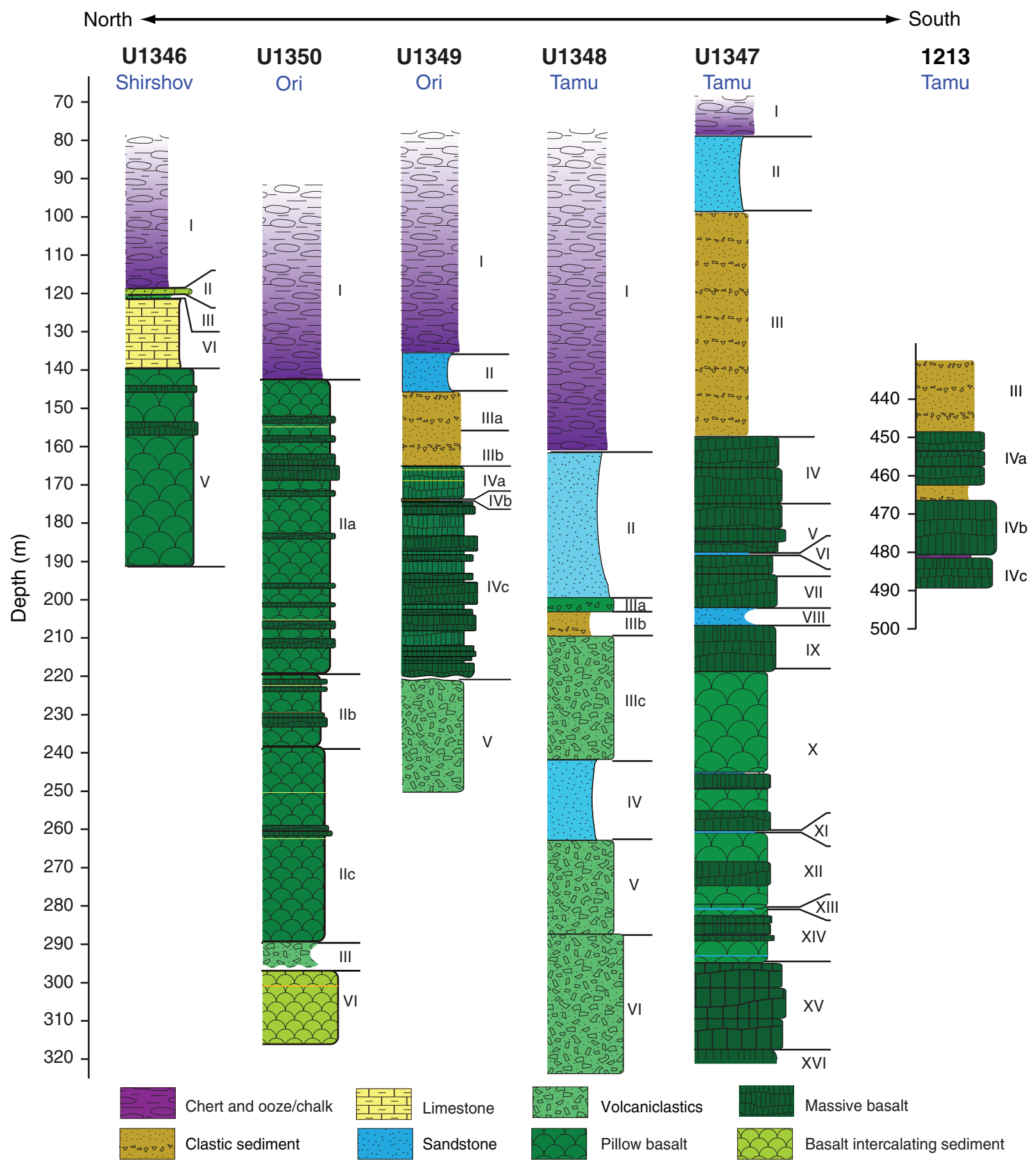


Figure F3. Alternating field (AF) demagnetization results (Sample 324-U1346A-6R-1, 83-85 cm). A. Equal angle spherical projection. B. Zijderveld plot. C. Magnetic intensity vs. demagnetization step. This sample exhibits higher median destructive field behavior typical of many AF demagnetized samples at site.

324-U1346A-6R-1, 83-85 cm

A

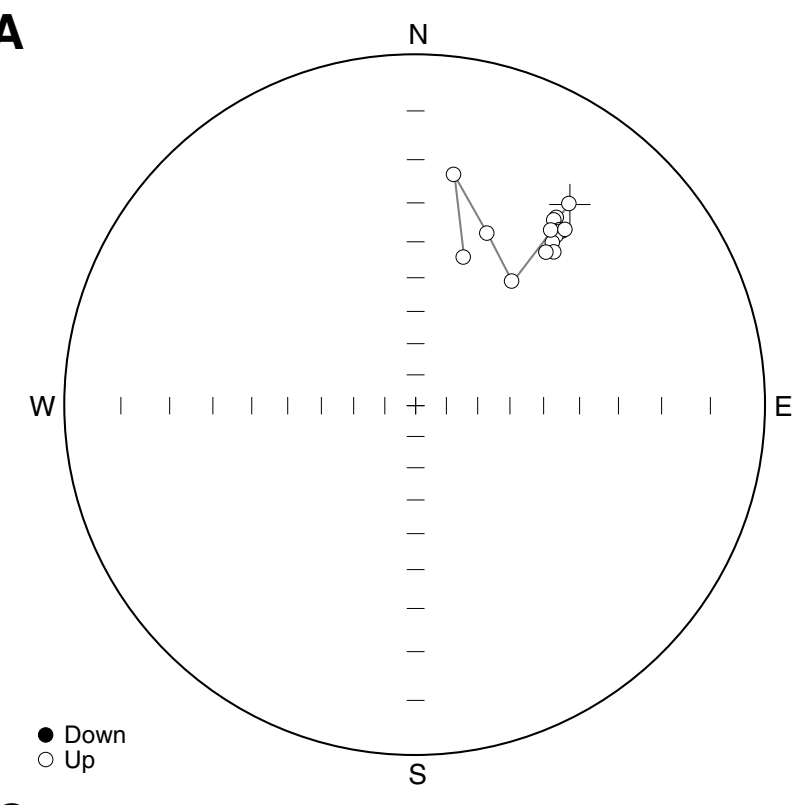

C

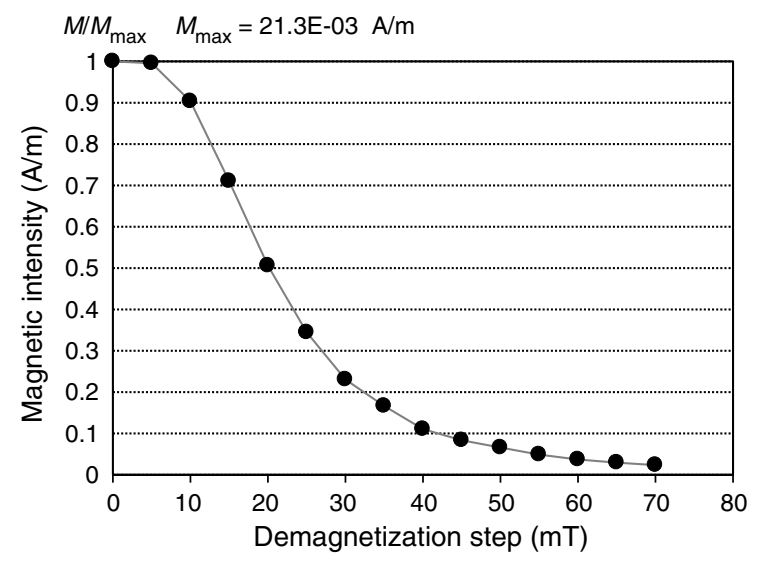

B

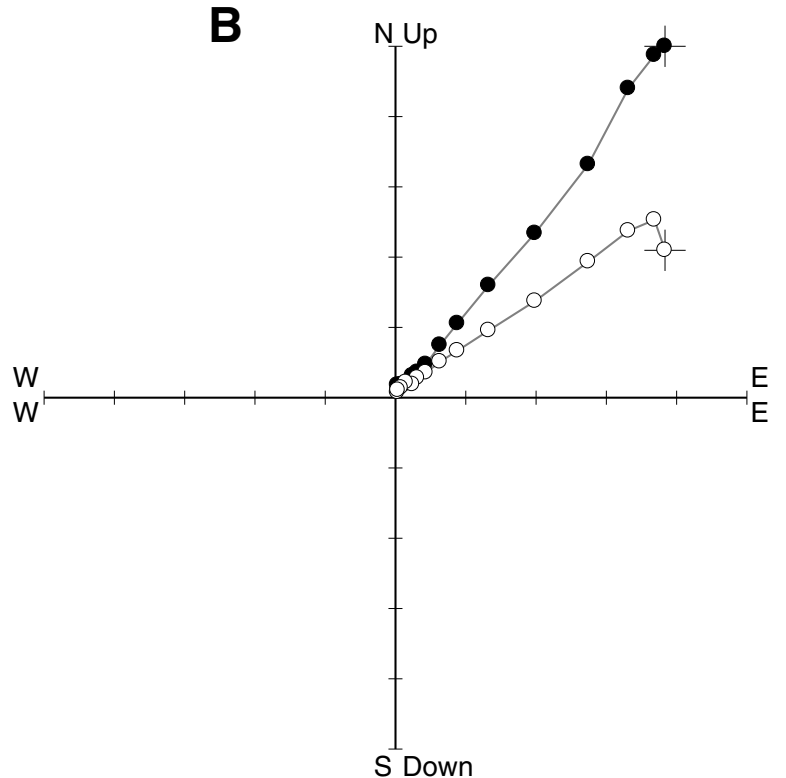

- Horizontal - - - Vertical
Unit $=3.20 \mathrm{E}-03 \mathrm{~A} / \mathrm{m}$ 
Figure F4. Alternating field demagnetization results (Sample 324-U1346A-10R-1, 40-42 cm). A. Equal angle spherical projection. B. Zijderveld plot. C. Magnetic intensity vs. demagnetization step. This sample displays low-median destructive field (MDF) behavior during demagnetization. Despite the low MDF, the weak magnetizations at higher demagnetization steps are consistent.

324-U1346A-10R-1, 40-42 cm

A

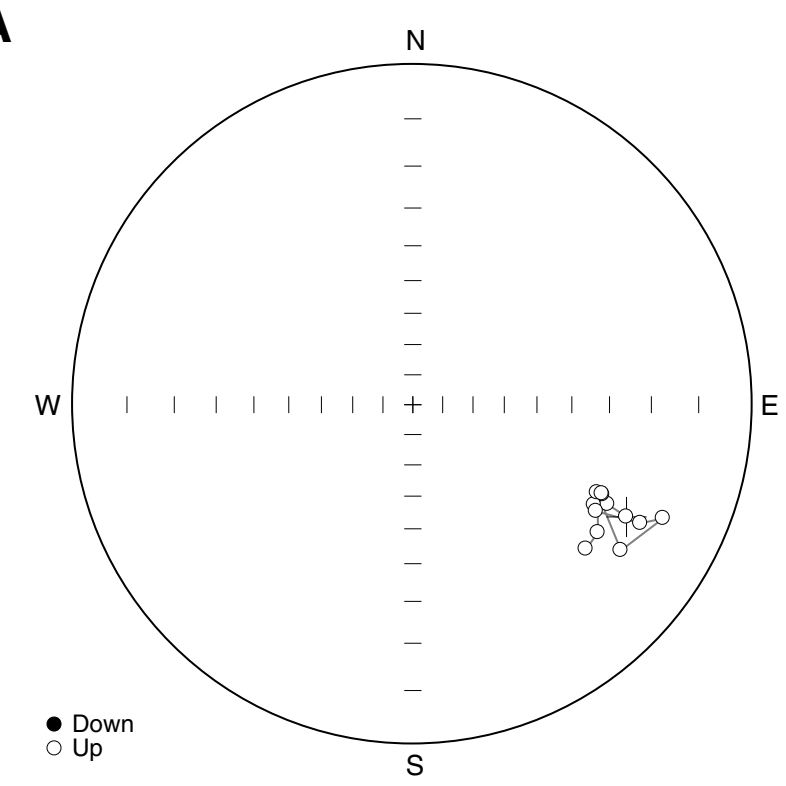

C

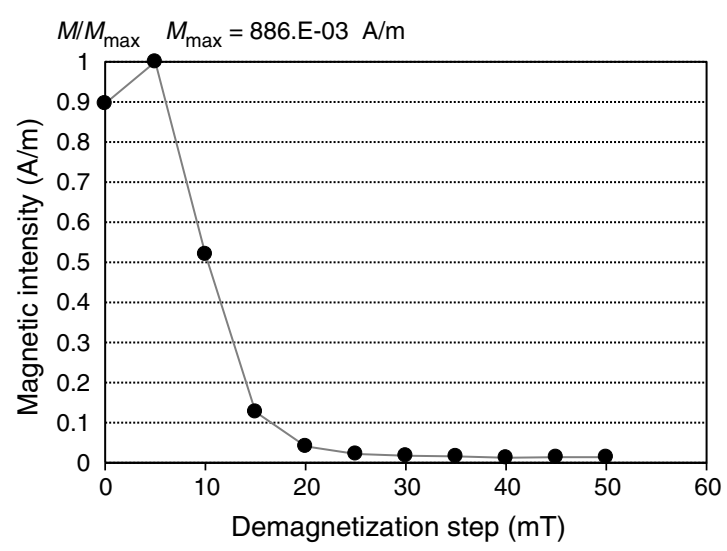

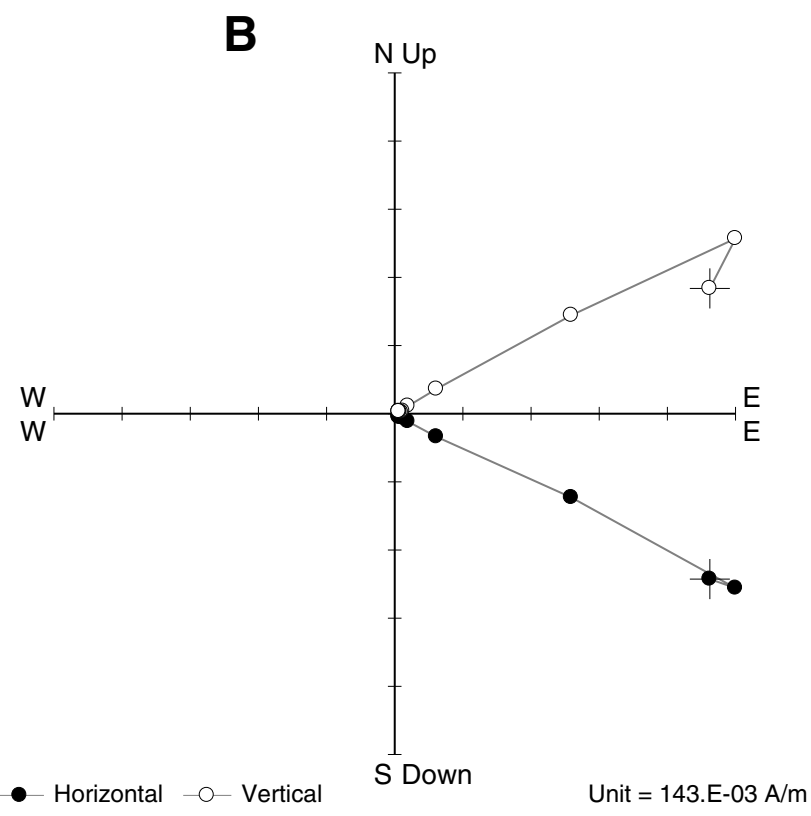


Figure F5. Thermal demagnetization results (Sample 324-U1346A-16R-2, 35-37 cm). A. Equal angle spherical projection. B. Zijderveld plot. C. Magnetic intensity vs. demagnetization temperature. This example shows behavior typical of thermally demagnetized samples at this site and a segment of partial self-reversal in the demagnetization path.

324-U1346A-16R-2, 35-37 cm

A

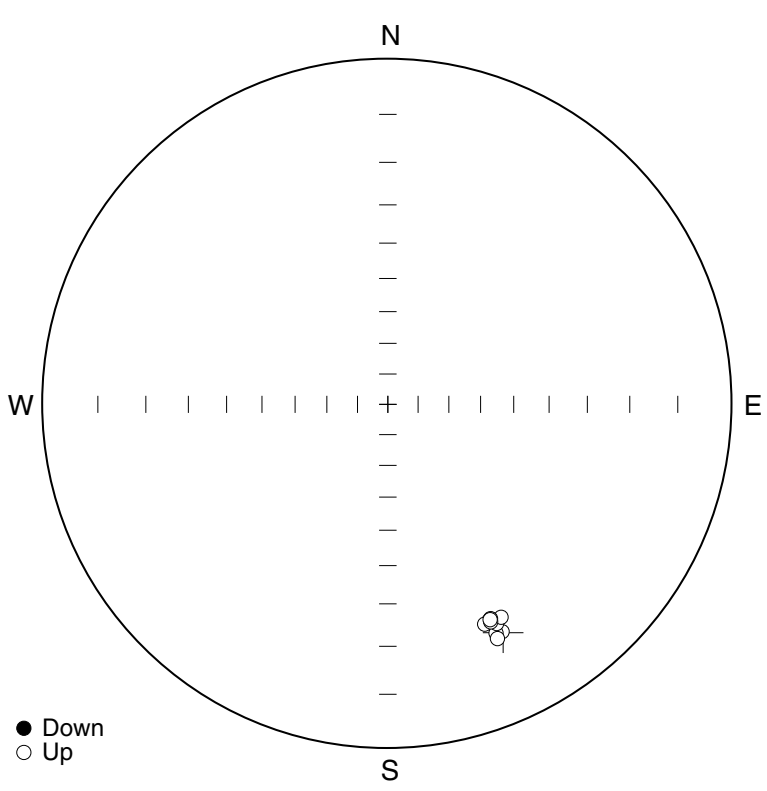

C

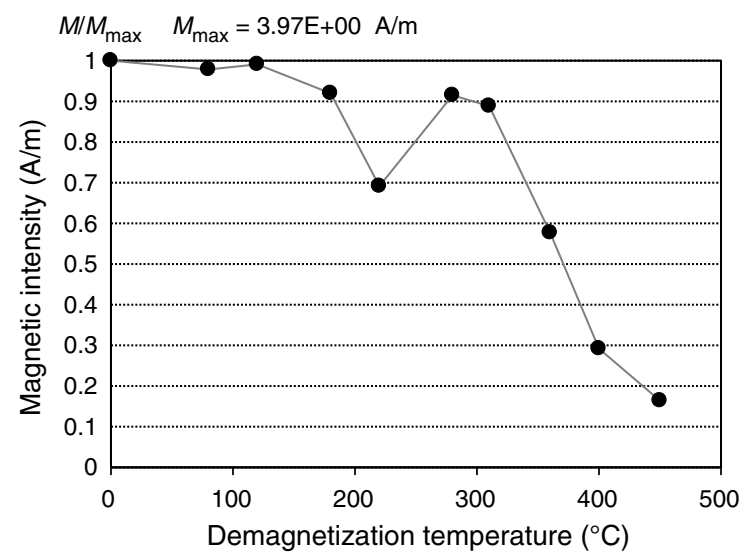

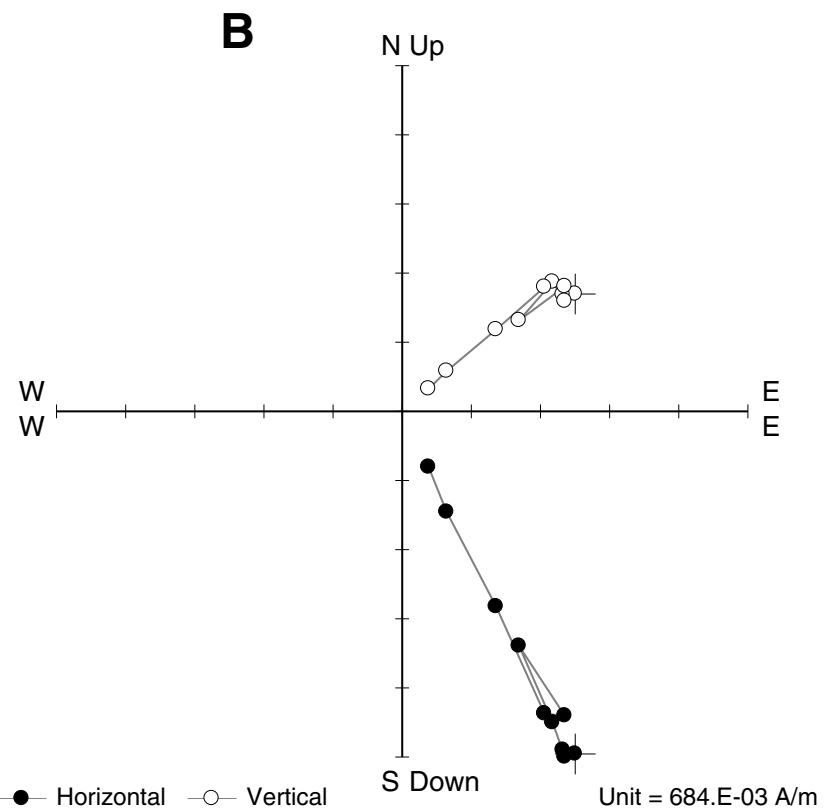

- Horizontal - $\_$- Vertical
Unit $=684 . \mathrm{E}-03 \mathrm{~A} / \mathrm{m}$ 
Figure F6. Plots of sample characteristic remanence inclinations vs. depth, Site U1346. AF = alternating field, WWU $=$ Northwest Paleomagnetism Laboratory at Western Washington University (USA).

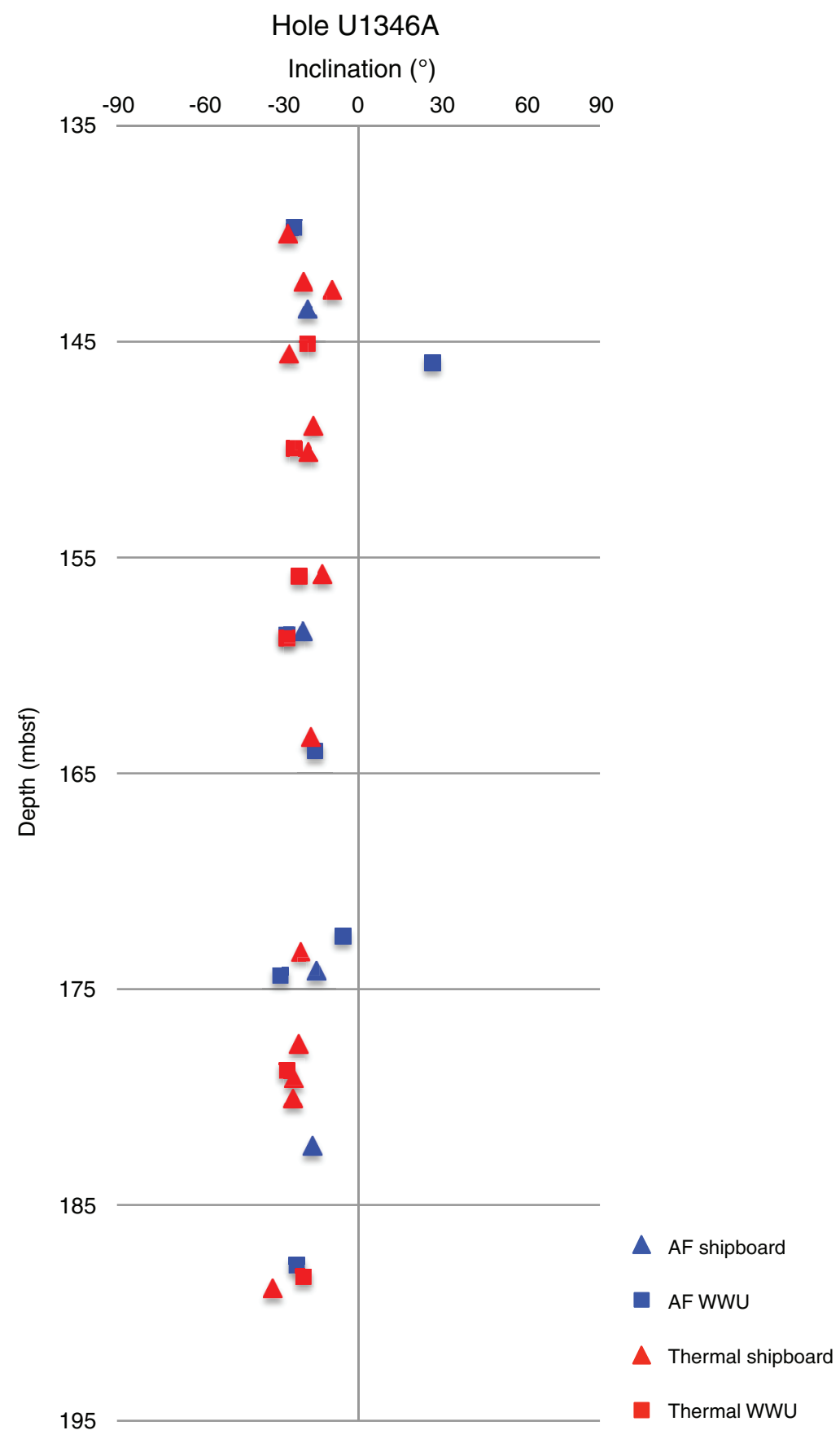


Figure F7. Alternating field (AF) demagnetization results (Sample 324-U1347A-13R-6, 132-134 cm). A. Equal angle spherical projection. B. Zijderveld plot. C. Magnetic intensity vs. demagnetization step. This sample exhibits higher median destructive field behavior during AF demagnetization.

324-U1347A-13R-6,132-134 cm

A

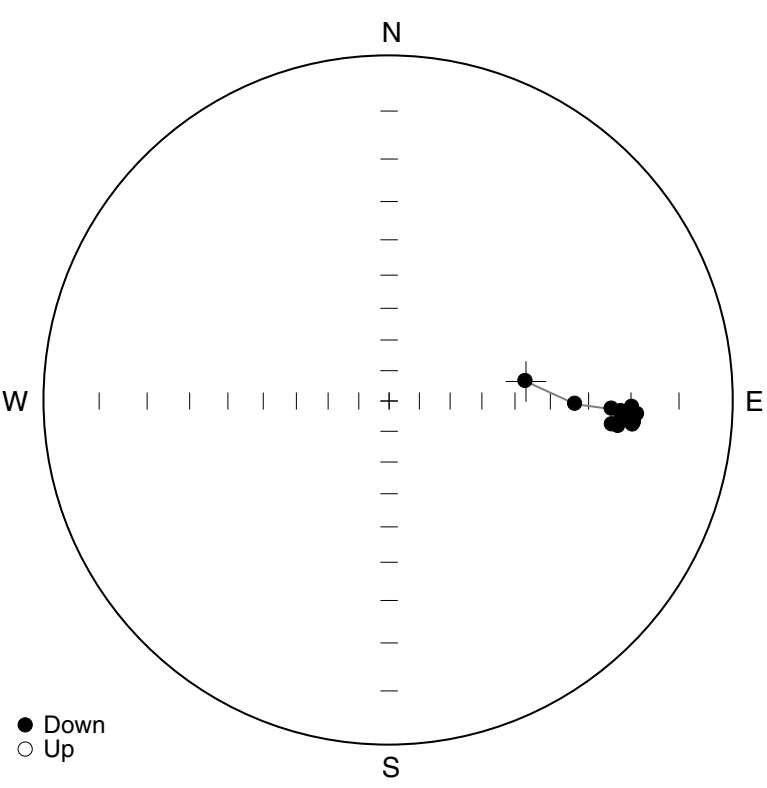

C

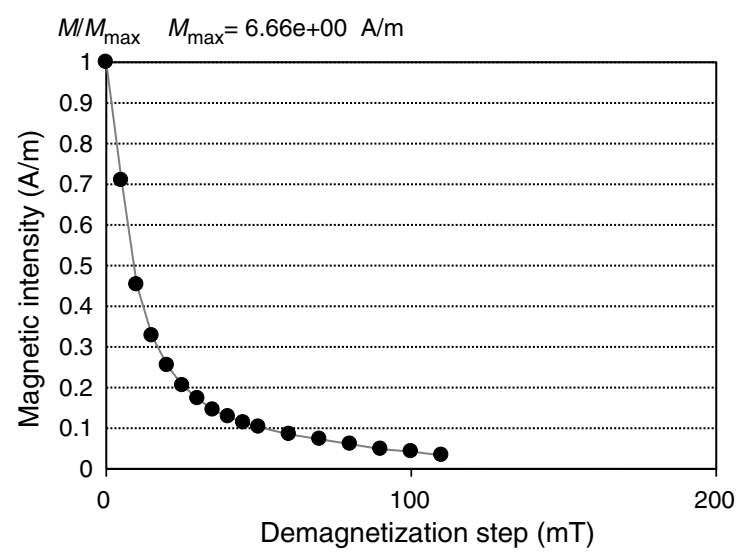

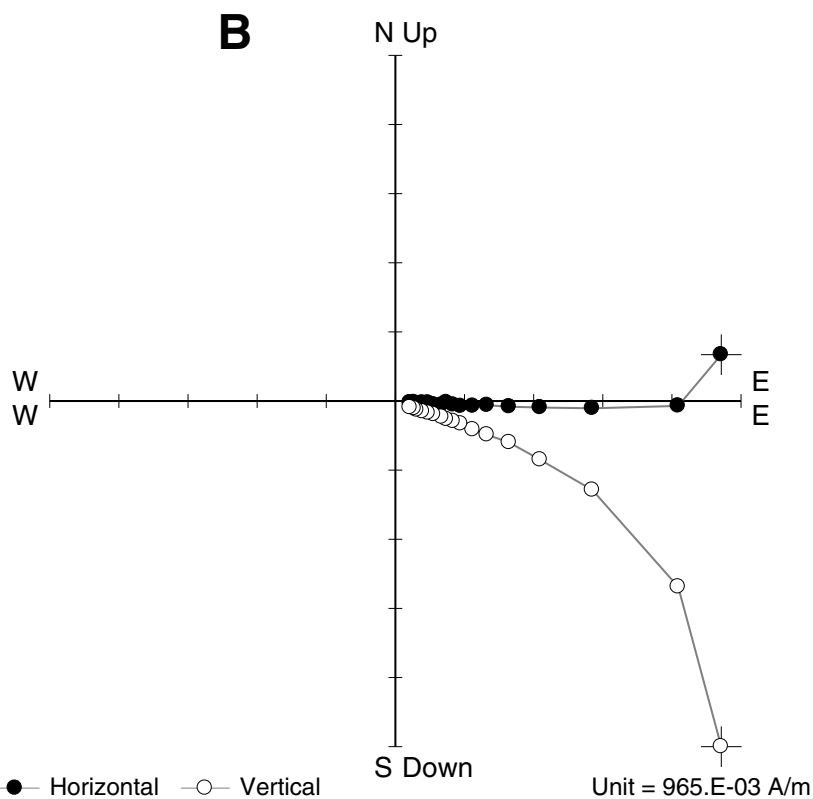

- Horizontal - - - Vertical 
Figure F8. Alternating field (AF) demagnetization results (Sample 324-U1347A-14R-1, 51-53 cm). A. Equal angle spherical projection. B. Zijderveld plot. C. Magnetic intensity vs. demagnetization step. This sample shows an example of low-median destructive field (MDF) behavior during AF demagnetization. Despite the low MDF, the weak magnetizations at higher demagnetization steps $<60 \mathrm{mT}$ are consistent.

324-U1347A-14R-1, 51-53 cm

A

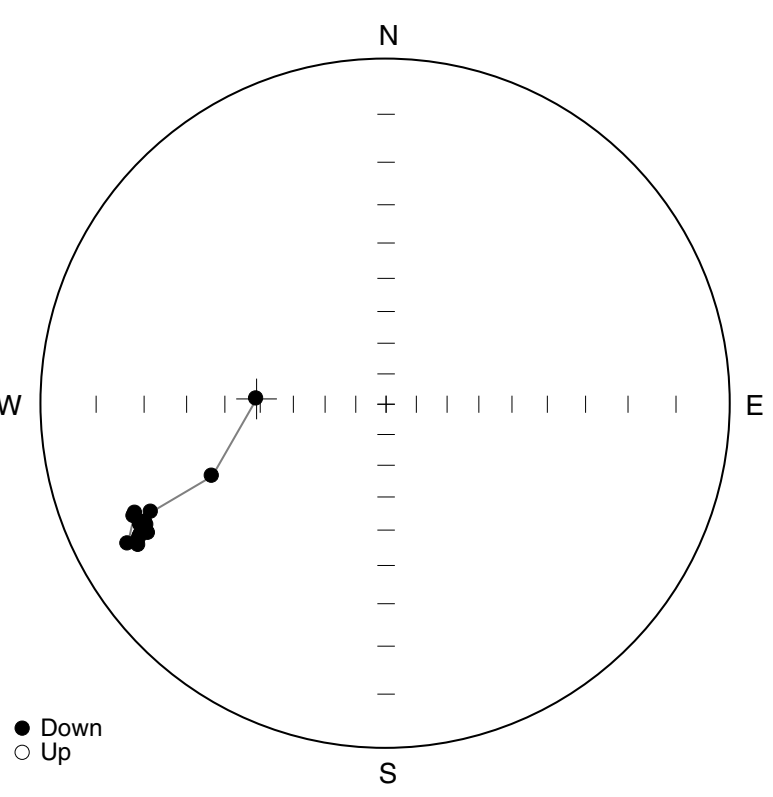

C

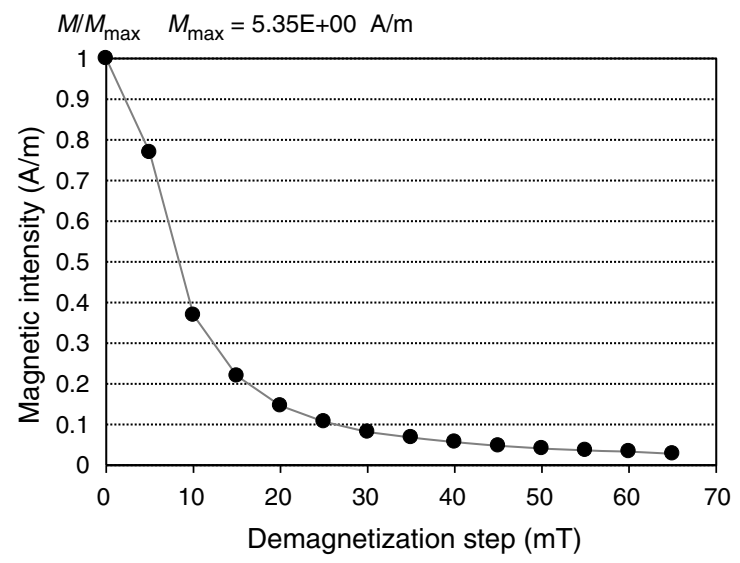

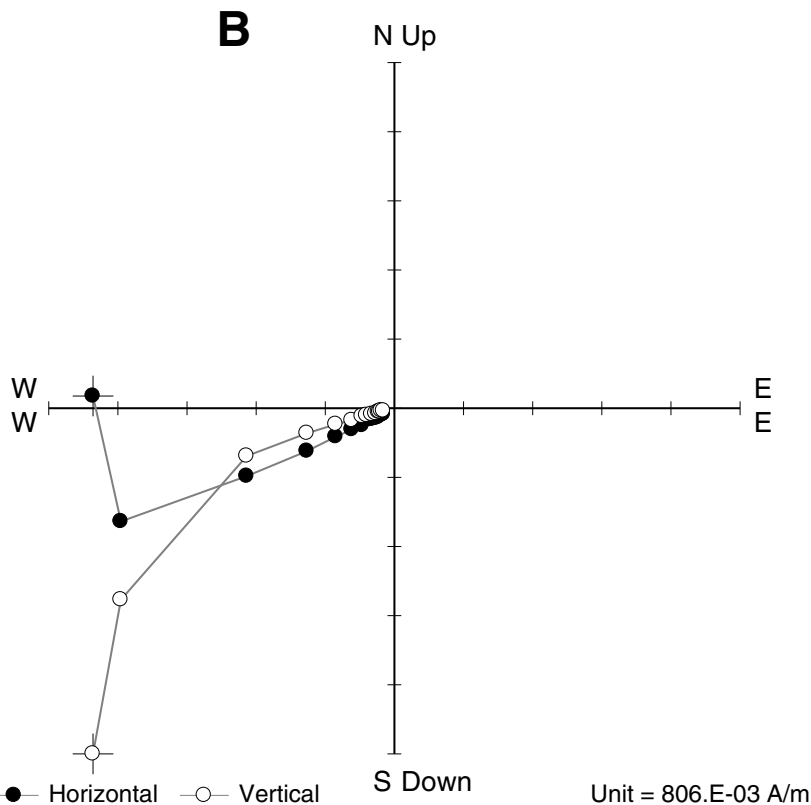


Figure F9. Thermal demagnetization results (Sample 324-U1347A-12R-2, 81-83 cm). A. Equal angle spherical projection. B. Zijderveld plot. C. Magnetic intensity vs. demagnetization temperature. This sample exhibits a linear decay of magnetization with increased heating. The last step at $575^{\circ} \mathrm{C}$ is inconsistent because sample was fully demagnetized at that temperature.

324-U1347A-12R-2, 81-83 cm

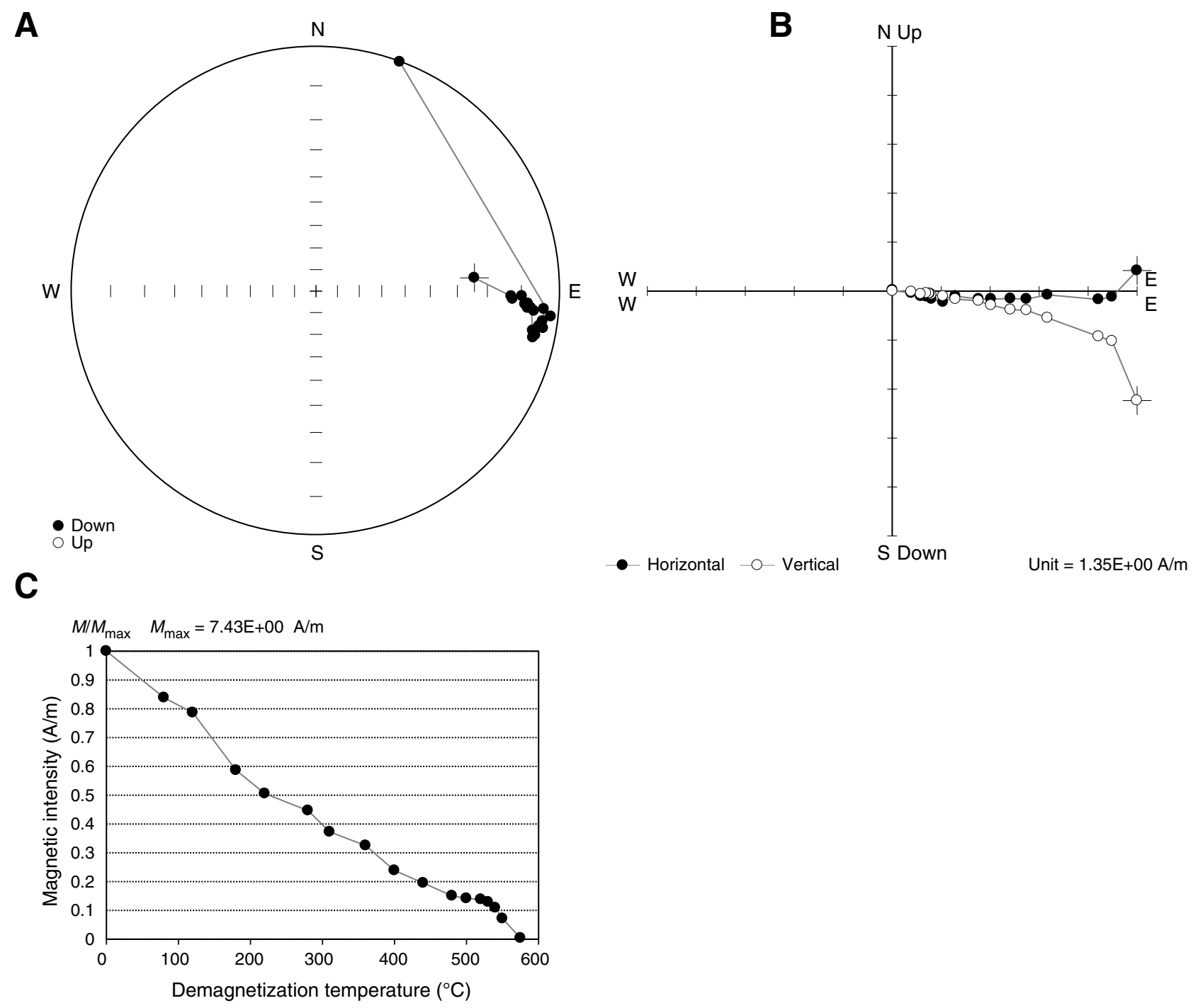


Figure F10. Thermal demagnetization results (Sample 324-U1347A-18R-3, 143-145 cm). A. Equal angle spherical projection. B. Zijderveld plot. C. Magnetic intensity vs. demagnetization temperature. This sample displays a significant drop in magnetization intensity at moderate heating steps.

324-U1347A-18R-3, 143-145 cm

A

A

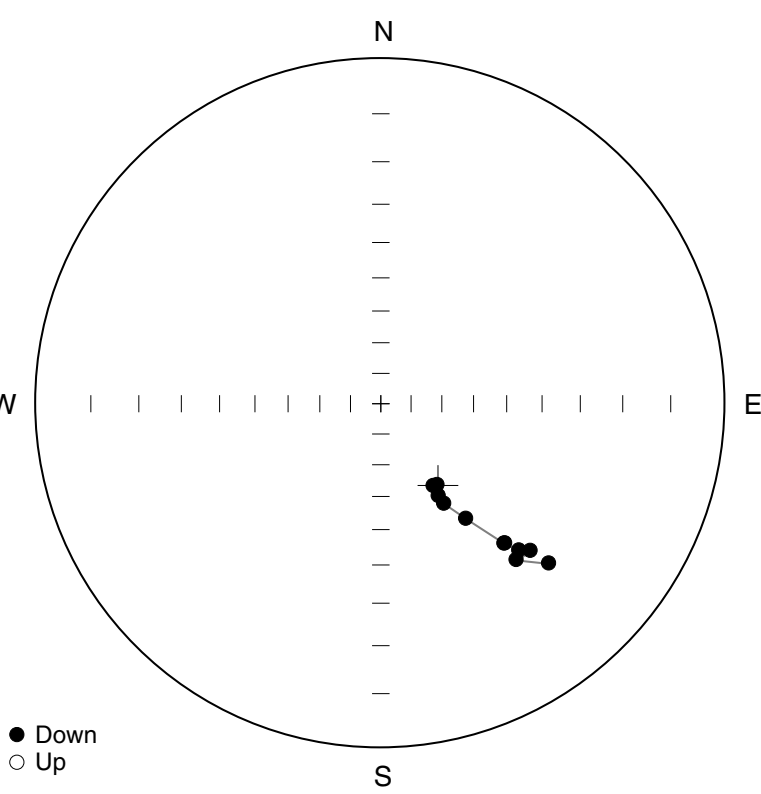

C

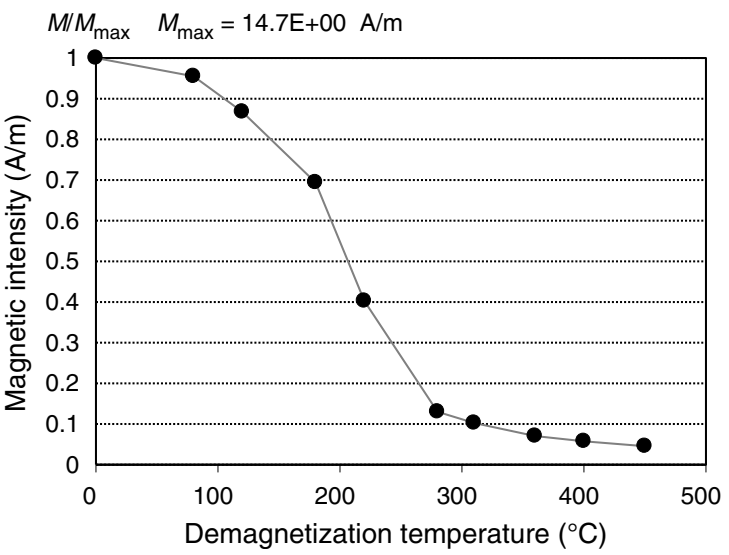

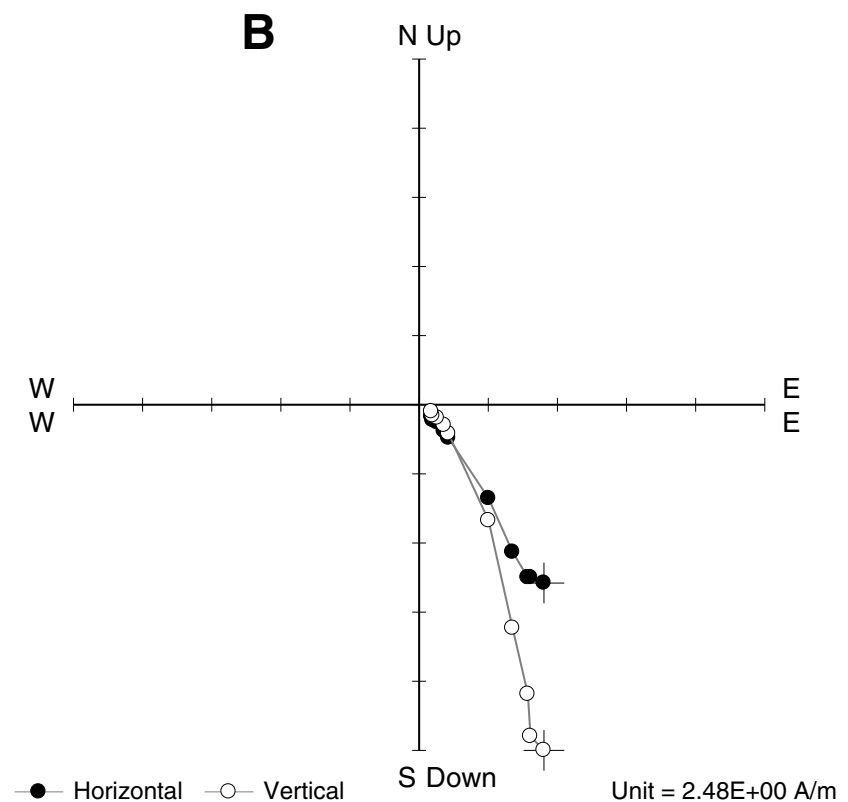


Figure F11. Thermal demagnetization results (Sample 324-U1347A-17R-2, 94-96 cm). A. Equal angle spherical projection. B. Zijderveld plot. C. Magnetic intensity vs. demagnetization step. This sample shows an example of a small self-reversal at moderate heating steps. Note that demagnetized magnetization directions are consistent through the self-reversed section except at $550^{\circ} \mathrm{C}$.

324-U1347A-17R-2, 94-96 cm

A

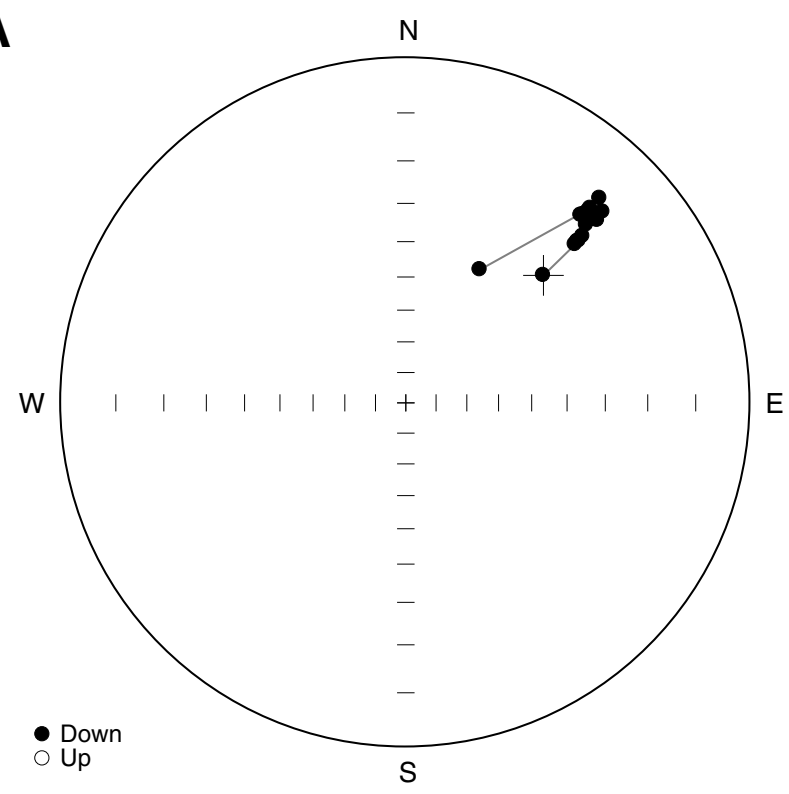

C

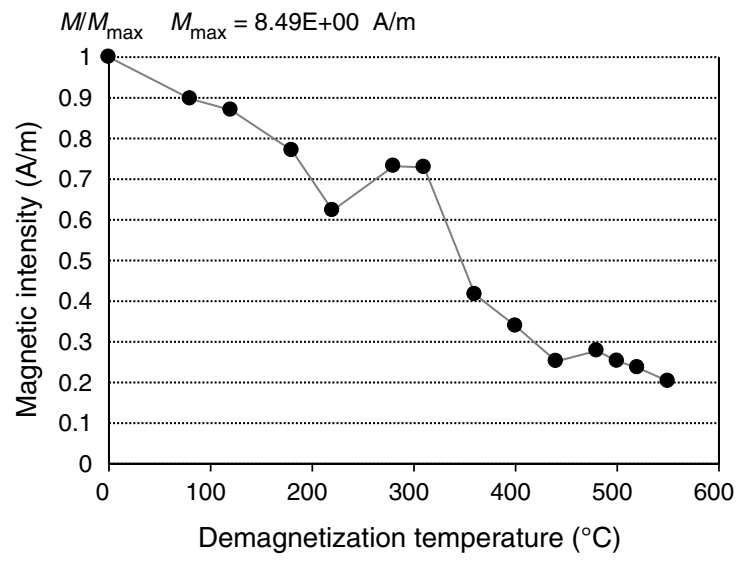

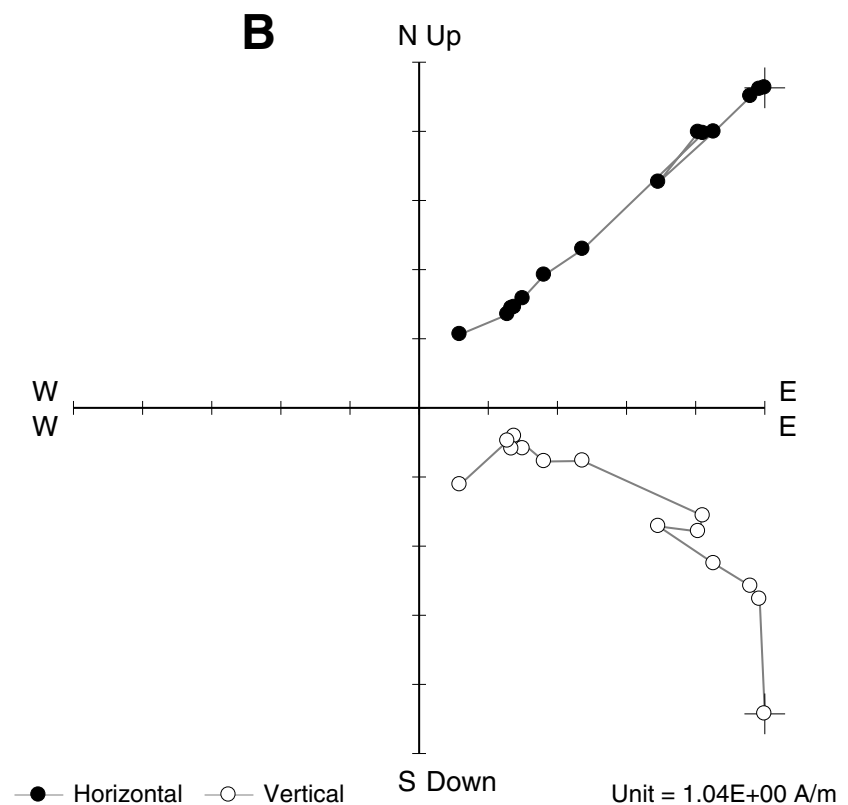


Figure F12. Plot of sample principal component inclinations vs. depth, Hole U1347A. AF = alternating field, WWU $=$ Northwest Paleomagnetism Laboratory at Western Washington University (USA).

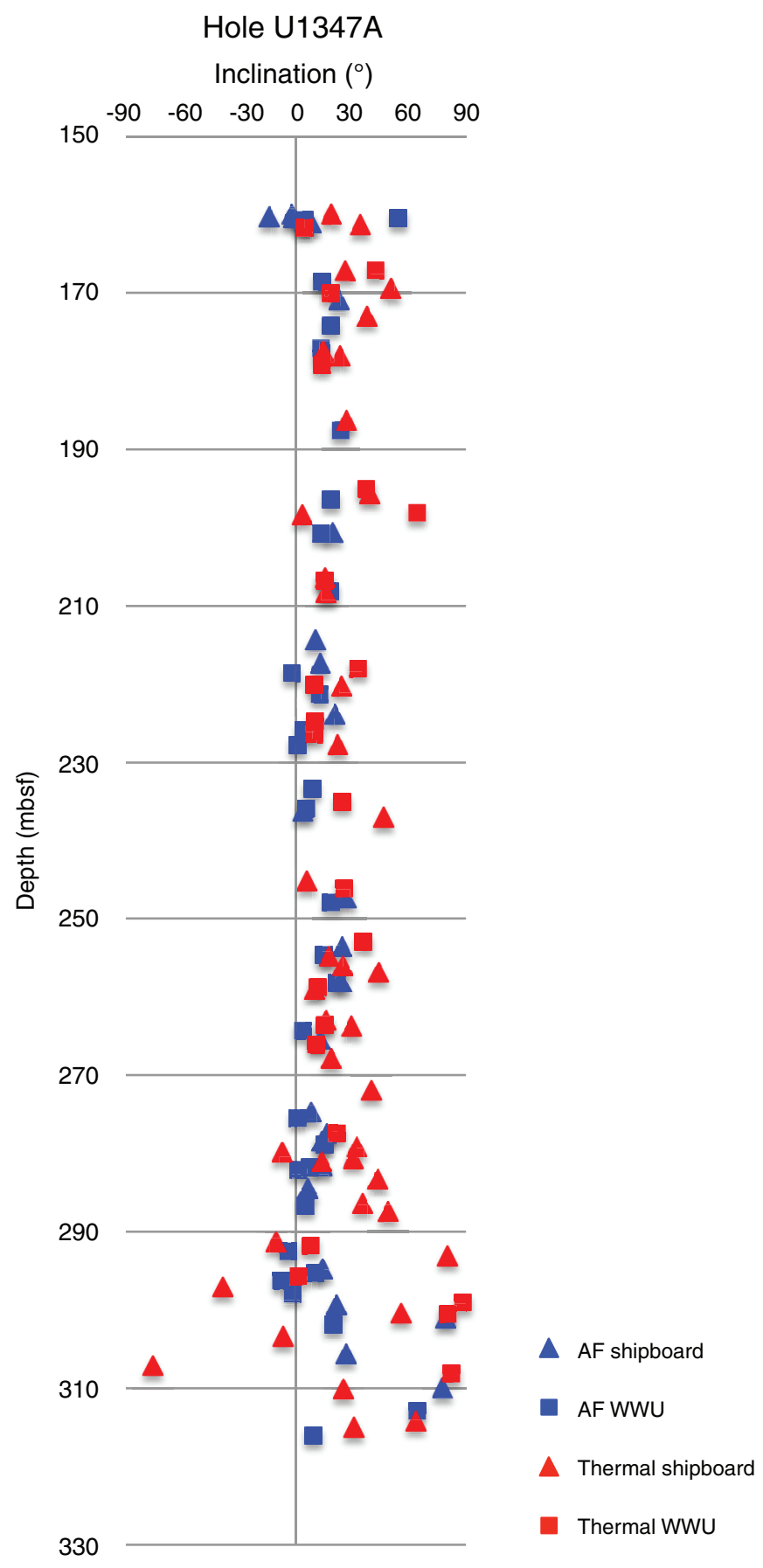


Figure F13. Alternating field (AF) demagnetization results (Sample 324-U1350A-17R-2, 80-82 cm). A. Equal angle spherical projection. B. Zijderveld plot. C. Magnetic intensity vs. demagnetization step. This sample exhibits low-median destructive field behavior during AF demagnetization. Once the overprint is removed, the inclination changes from positive to negative.

324-U1350A-17R-2, 80-82 cm

A

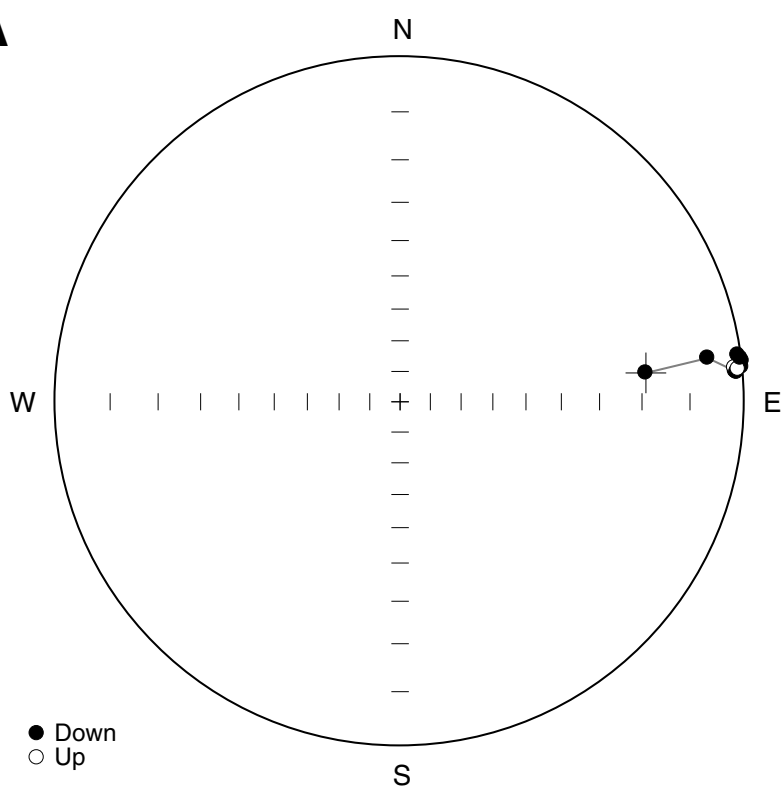

C

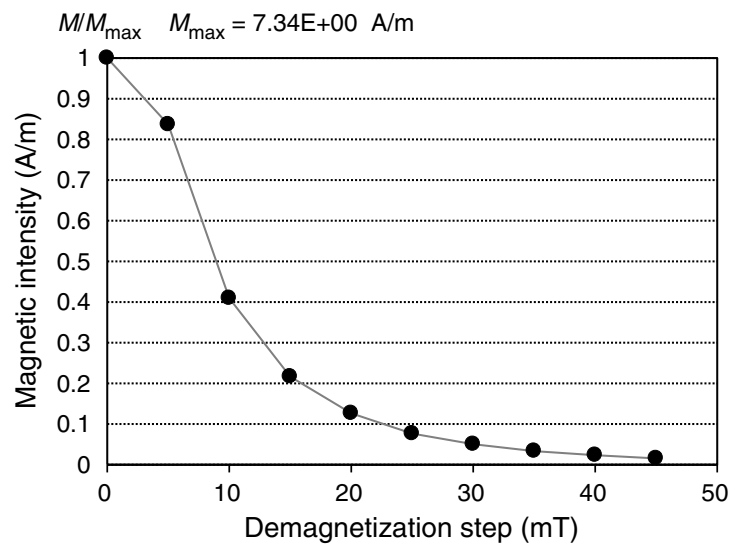

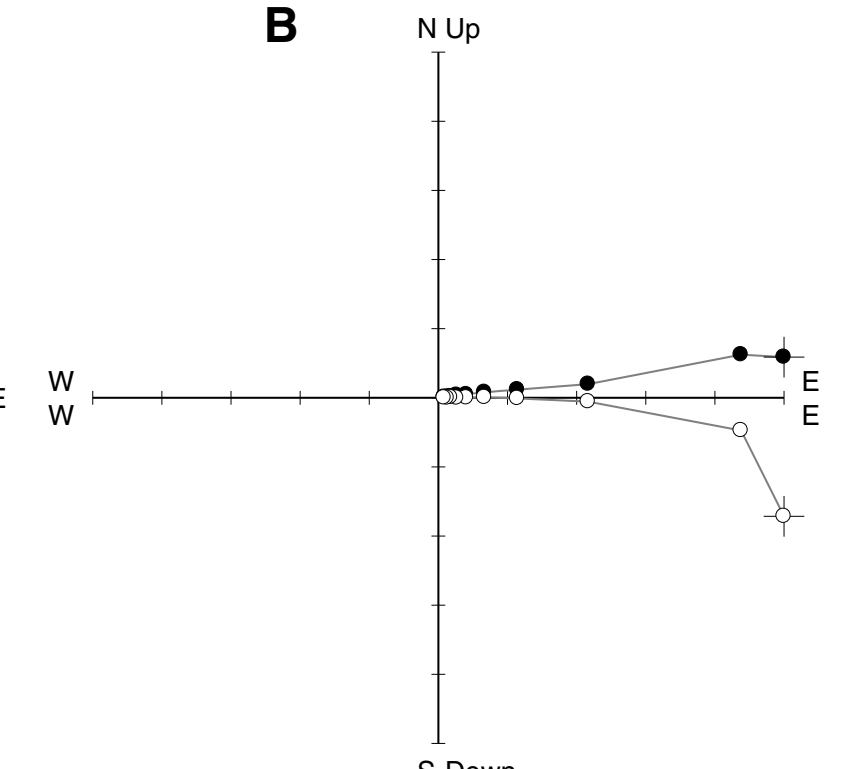

- Horizontal - - - Vertical
S Down

Unit $=1.38 \mathrm{E}+00 \mathrm{~A} / \mathrm{m}$ 
Figure F14. Alternating field (AF) demagnetization results (Sample 324-U1347A-24R-2, 137-139 cm). A. Equal angle spherical projection. B. Zijderveld plot. C. Magnetic intensity vs. demagnetization step. This sample displays higher MDF behavior during AF demagnetization.

324-U1347A-24R-2, 137-139 cm

A

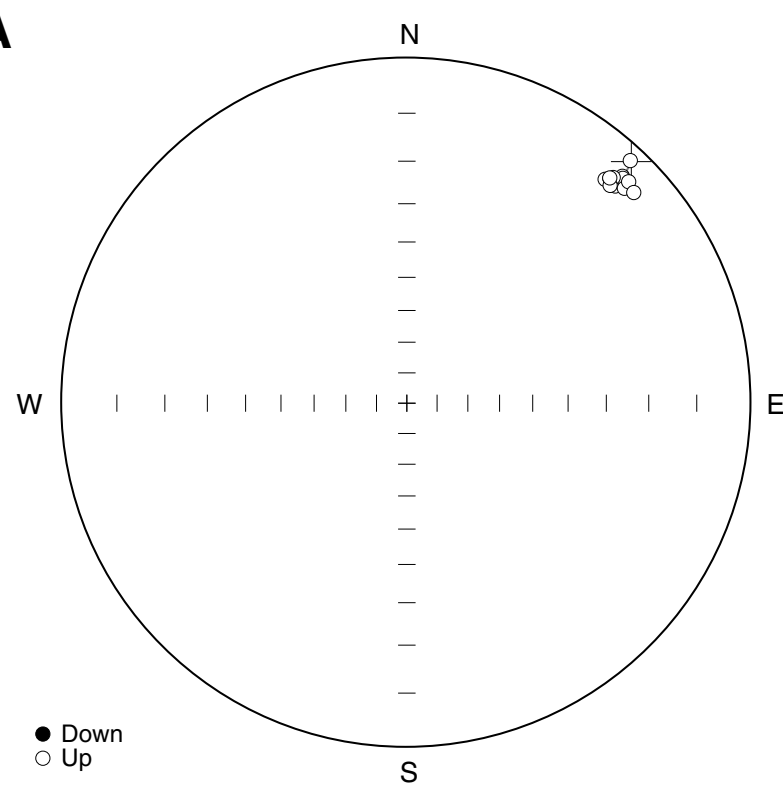

C

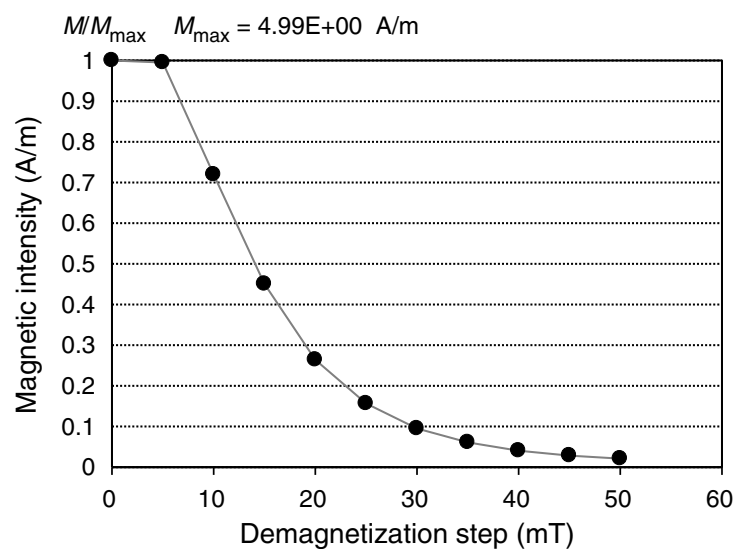

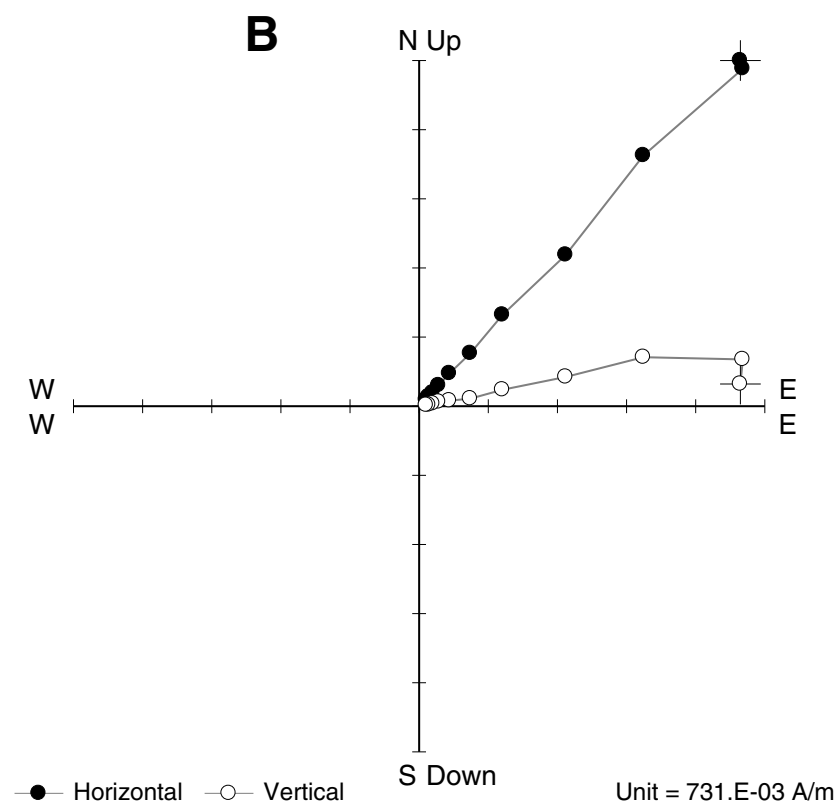

- Horizontal _o- Vertical 
Figure F15. Thermal demagnetization results (Sample 324-U1347A-23R-4, 16-18 cm). A. Equal angle spherical projection. B. Zijderveld plot. C. Magnetic intensity vs. demagnetization temperature. This sample shows linear decay of magnetization intensity with increased temperature.

\section{4-U1347A-23R-4, 16-18 cm}

A

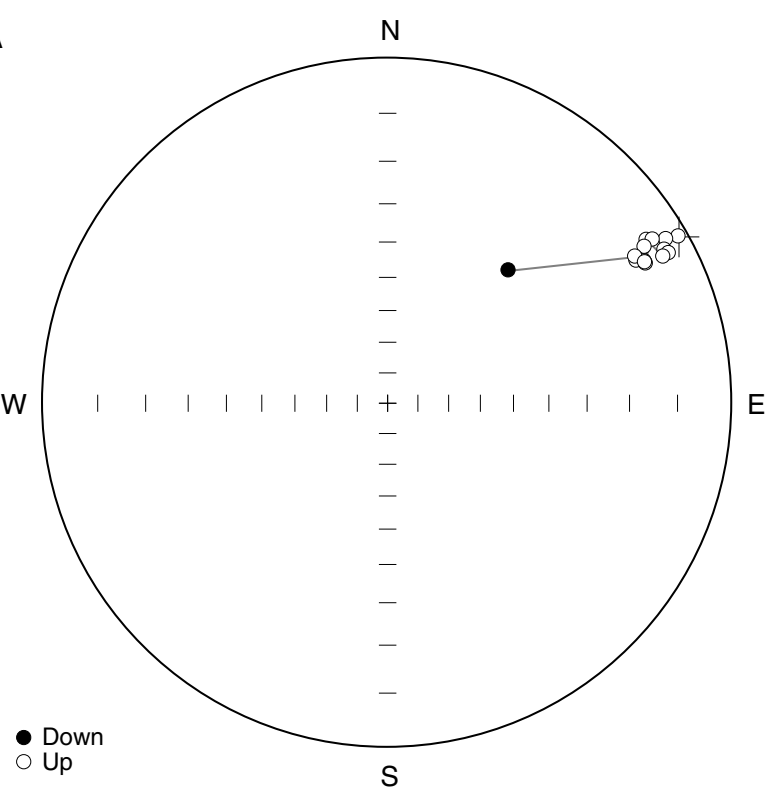

C

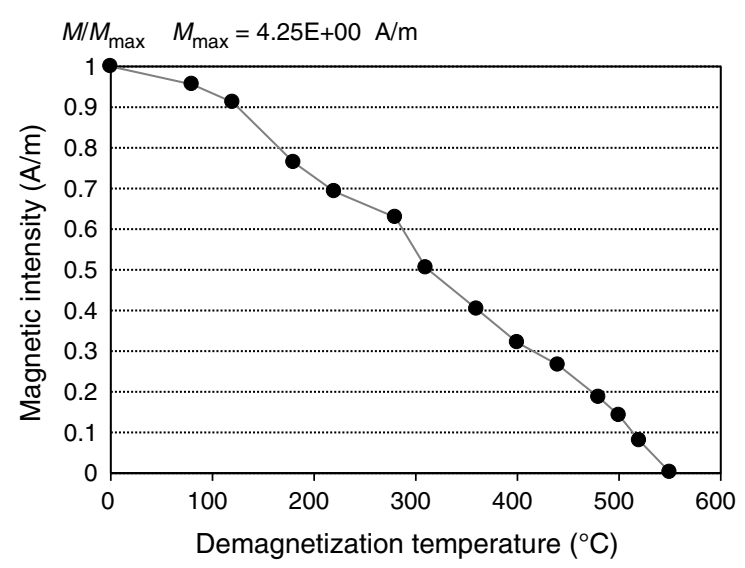

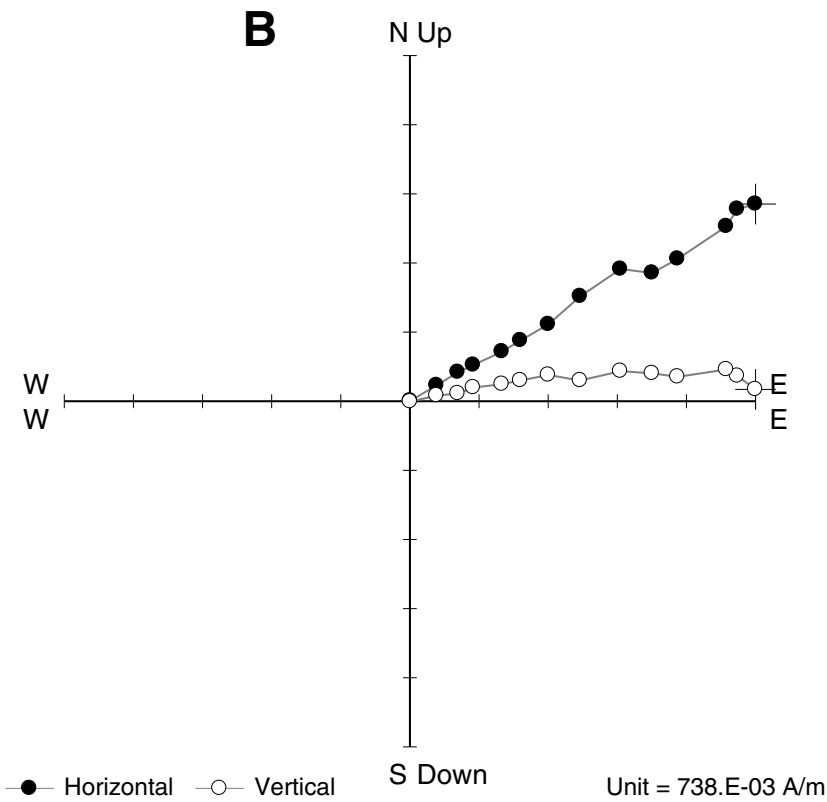


Figure F16. Thermal demagnetization results (Sample 324-U1347A-21R-2, 135-137 cm). A. Equal angle spherical projection. B. Zijderveld plot. C. Magnetic intensity vs. demagnetization step. This sample displays a small self-reversal during thermal demagnetization at moderate temperature steps.
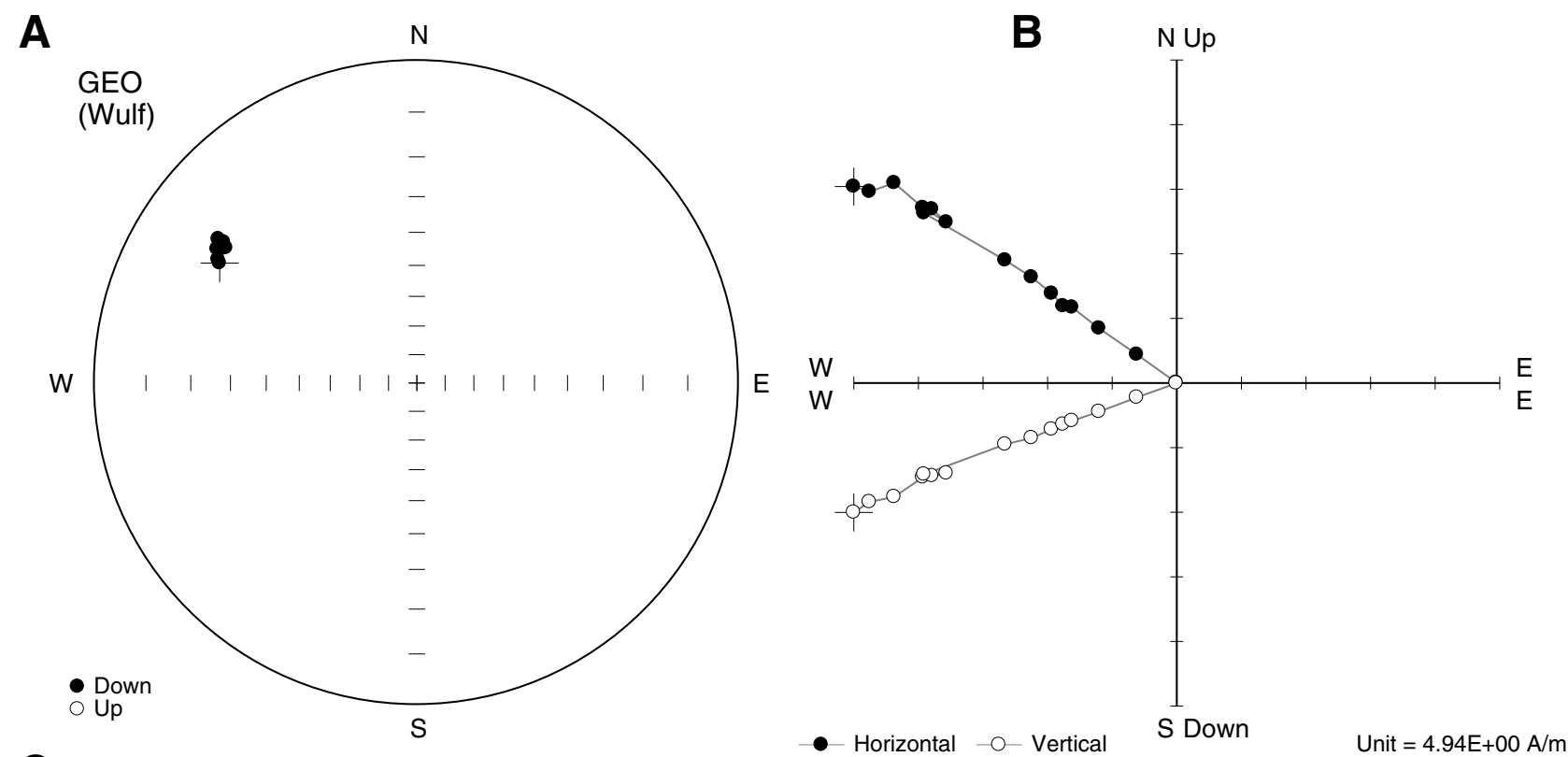

C

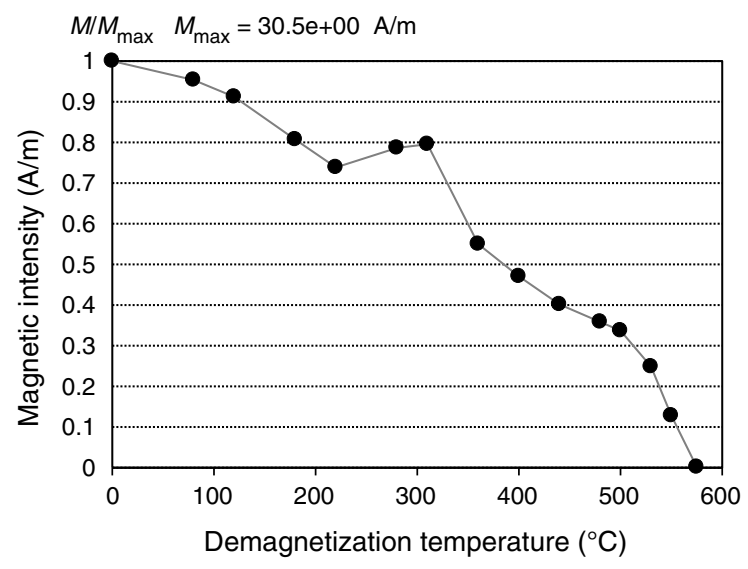


Figure F17. Plot of sample principal component inclinations vs. depth, Hole U1350A. AF = alternating field, WWU $=$ Northwest Paleomagnetism Laboratory at Western Washington University (USA).

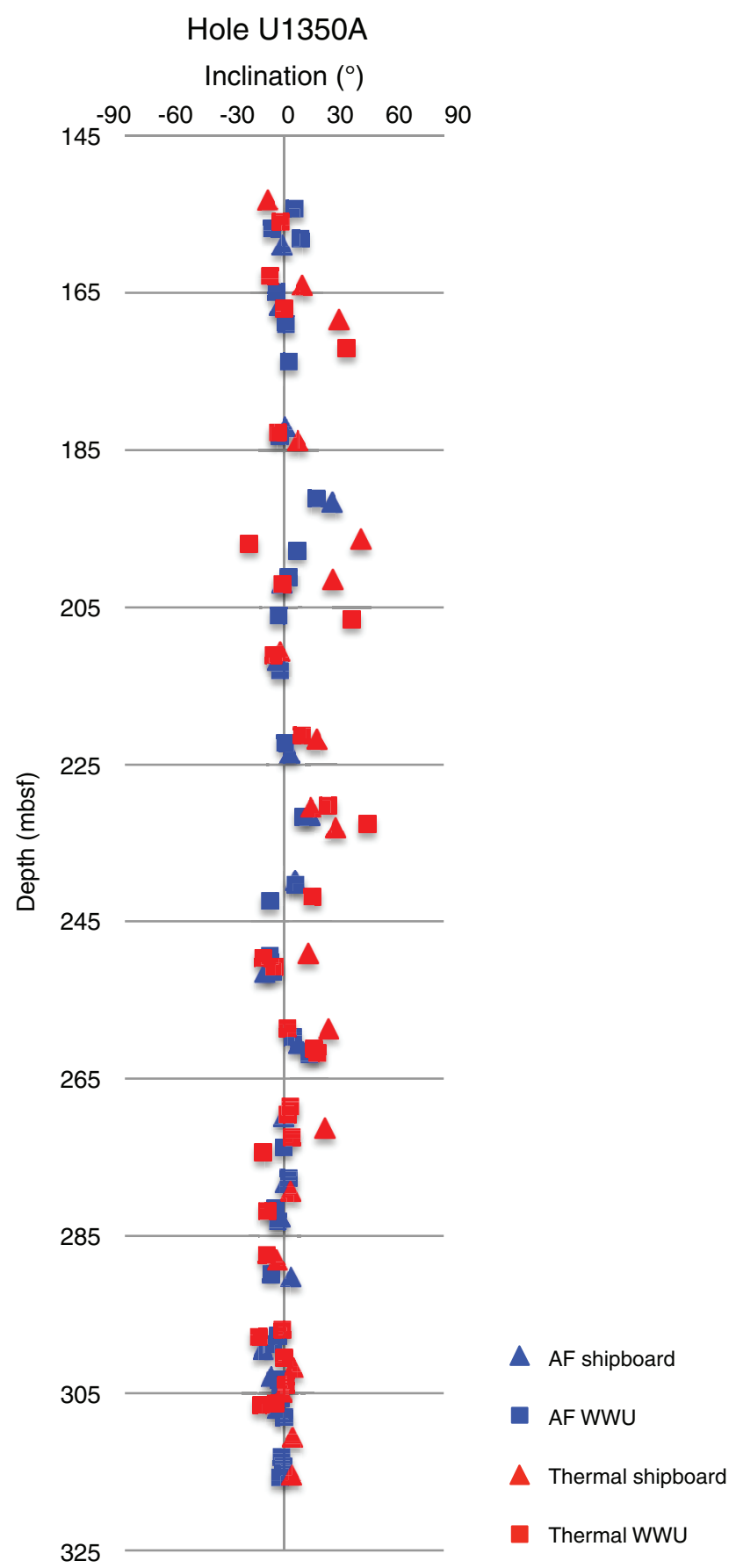


Figure F18. Hysteresis loops for Site U1346 samples.
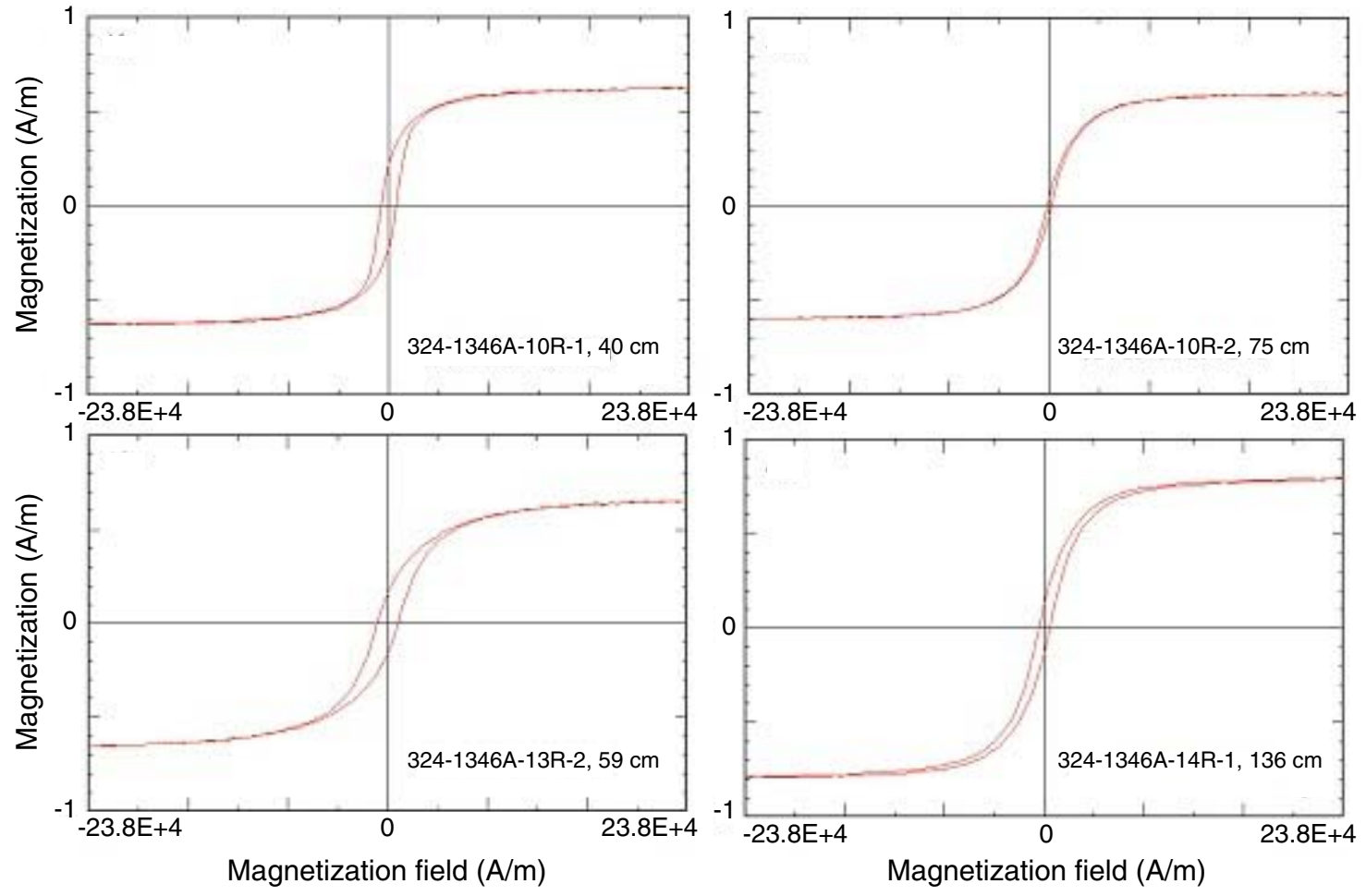
Figure F19. Hysteresis loops for Site U1347 samples.
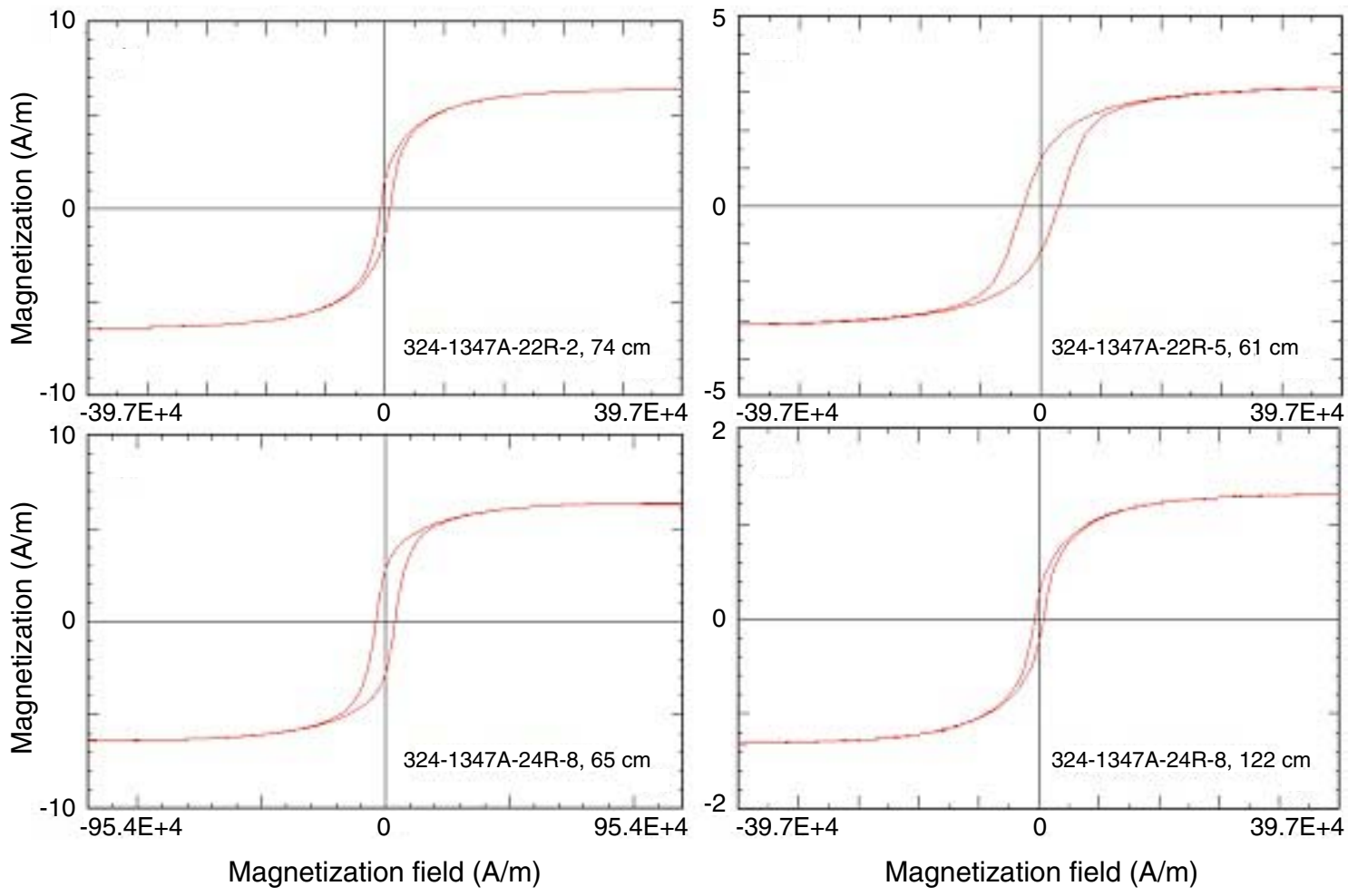
Figure F20. Hysteresis loops for Site U1350 samples.
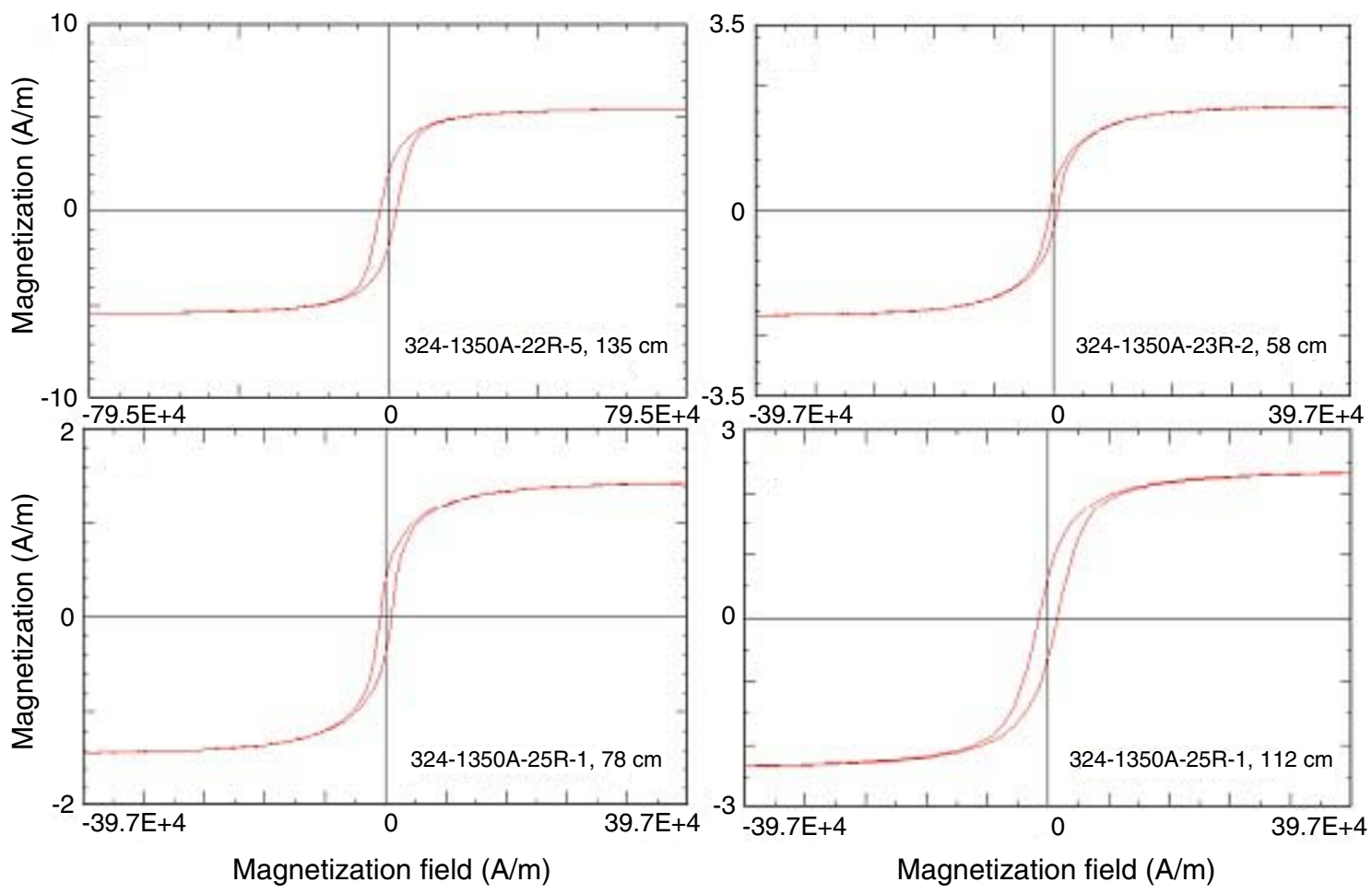
Figure F21. Day plot (Day et al., 1977) for Expedition 324 basalt samples. SD = single domain, PSD = pseudosingle domain. $\mathrm{MD}+\mathrm{SPM}=$ multidomain and superparamagnetic.

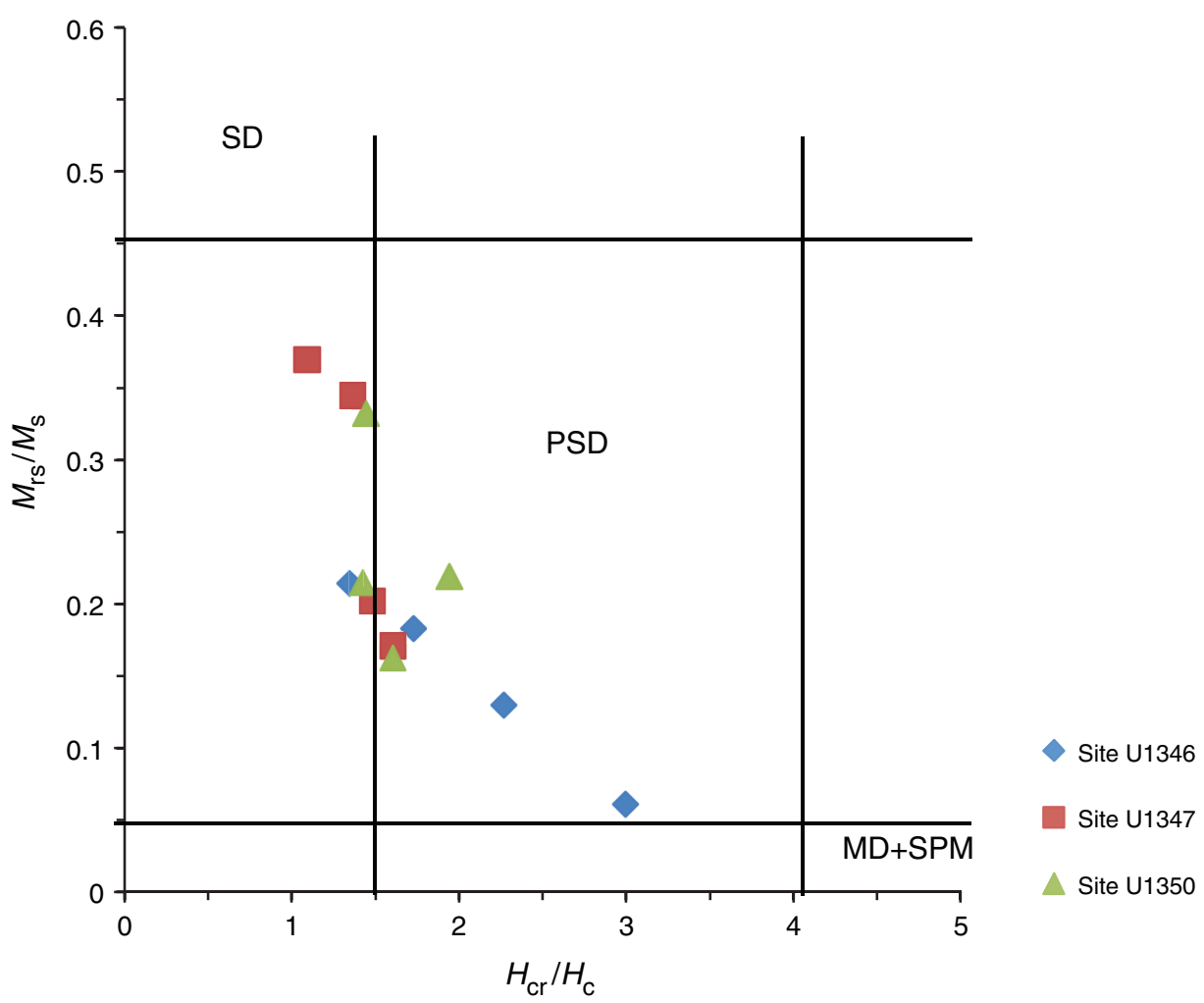


Table T1. Discrete sample paleomagnetic results, Hole U1346A.

\begin{tabular}{|c|c|c|c|c|c|c|c|c|c|c|c|}
\hline $\begin{array}{l}\text { Core, section, } \\
\text { interval }(\mathrm{cm})\end{array}$ & $\begin{array}{l}\text { Depth } \\
\text { (mbsf) }\end{array}$ & $\begin{array}{l}\text { Flow } \\
\text { unit }\end{array}$ & Lithology & $\begin{array}{l}\text { Analysis } \\
\text { location }\end{array}$ & $\begin{array}{l}\text { Demagnetization } \\
\text { treatment }\end{array}$ & $\begin{array}{l}\text { Steps } \\
\left(\mathrm{mT},{ }^{\circ} \mathrm{C}\right)\end{array}$ & $N$ & $\begin{array}{c}\text { Inc anc } \\
\left({ }^{\circ}\right)\end{array}$ & $\begin{array}{c}\text { MAD anc } \\
\left({ }^{\circ}\right)\end{array}$ & $\begin{array}{l}\text { Inc } \\
\left({ }^{\circ}\right)\end{array}$ & $\begin{array}{l}\text { MAD } \\
\left({ }^{\circ}\right)\end{array}$ \\
\hline \multicolumn{12}{|l|}{ 324-U1346A- } \\
\hline $6 \mathrm{R}-1,83-85$ & 139.73 & 19 & Highly vesicular basalt & WWU & $\mathrm{AF}$ & $25-60$ & 8 & -24.0 & 3.0 & -23.4 & 2.8 \\
\hline $6 \mathrm{R}-1,111-113$ & 140.01 & 20 & Vesicular aphyric basalt & Shipboard & $\mathrm{TH}$ & $300-475$ & 6 & -26.4 & 1.7 & & \\
\hline 7R-1, 53.5-55.5 & 142.24 & 22 & Aphyric amygdaloidal pillow basalt & Shipboard & $\mathrm{TH}$ & $300-475$ & 6 & -20.5 & 2.4 & & \\
\hline 7R-1, 92-94 & 142.62 & 23 & Aphyric amygdaloidal pillow basalt & Shipboard & $\mathrm{TH}$ & $300-425$ & 4 & -9.8 & 2.3 & & \\
\hline $7 \mathrm{R}-2,39-41$ & 143.50 & 23 & Aphyric amygdaloidal pillow basalt & Shipboard & $\mathrm{AF}$ & $10-60$ & 8 & -19.0 & 0.8 & & \\
\hline $7 R-3,49-51$ & 145.10 & 24 & Aphyric amygdaloidal pillow basalt & WWU & $\mathrm{TH}$ & $310-450$ & 4 & -18.9 & 1.9 & -18.6 & 2.4 \\
\hline $7 R-4,12-14$ & 145.59 & 26 & Aphyric amygdaloidal pillow basalt & Shipboard & $\mathrm{TH}$ & $300-425$ & 4 & -25.7 & 1.5 & & \\
\hline $7 \mathrm{R}-4,53-55$ & 146.00 & 26 & Aphyric amygdaloidal pillow basalt & WWU & $\mathrm{AF}$ & $20-50$ & 7 & 27.6 & 1.1 & 27.7 & 1.2 \\
\hline $8 \mathrm{R}-1,136-138$ & 149.96 & 31 & Vesicular aphyric basalt & WWU & $\mathrm{TH}$ & $280-450$ & 5 & -24.0 & 2.2 & -23.7 & 2.5 \\
\hline $8 \mathrm{R}-2,11-13$ & 150.13 & 31 & Vesicular aphyric basalt & Shipboard & $\mathrm{TH}$ & $200-525$ & 9 & -18.9 & 3.0 & & \\
\hline 9R-1, 104.5-106.5 & 155.88 & 32 & Vesicular/amygdaloidal aphyric basalt & WWU & $\mathrm{TH}$ & $220-450$ & 6 & -21.9 & 4.9 & -21.1 & 6.1 \\
\hline $9 \mathrm{R}-2,95-98$ & 155.78 & 32 & Vesicular/amygdaloidal aphyric basalt & Shipboard & $\mathrm{TH}$ & $300-450$ & 5 & -13.4 & 3.3 & & \\
\hline $10 \mathrm{R}-1,21.5-23.5$ & 158.42 & 34 & Aphyric amygdaloidal basalt & Shipboard & $\mathrm{AF}$ & 10-100 & 11 & -20.8 & 3.6 & & \\
\hline $10 \mathrm{R}-1,40-42$ & 158.60 & 34 & Aphyric amygdaloidal basalt & WWU & $\mathrm{AF}$ & $10-50$ & 9 & -26.9 & 1.1 & -26.9 & 1 \\
\hline 10R-2, 75-77 & 158.74 & 35 & Aphyric amygdaloidal basalt & WWU & $\mathrm{TH}$ & $310-440$ & 4 & -26.5 & 6.0 & -26.2 & 6.4 \\
\hline $11 \mathrm{R}-1,35-37$ & 163.35 & 36 & Aphyric pillow basalt & Shipboard & $\mathrm{TH}$ & $300-450$ & 6 & -17.7 & 6.2 & & \\
\hline 11R-1, 97-99 & 163.97 & 37 & Aphyric pillow basalt & WWU & $\mathrm{AF}$ & $15-55$ & 9 & -16.1 & 1.8 & -15.8 & 1.8 \\
\hline $13 R-1,5-7$ & 172.55 & 37 & Aphyric pillow basalt & WWU & $\mathrm{AF}$ & $25-50$ & 6 & -5.9 & 2.9 & -4 & 1.7 \\
\hline $13 R-1,76-78$ & 173.26 & 38 & Aphyric amygdaloidal pillow basalt & Shipboard & $\mathrm{TH}$ & $300-500$ & 8 & -21.5 & 3.0 & & \\
\hline $13 R-2,38-41$ & 174.15 & 39 & Aphyric amygdaloidal pillow basalt & Shipboard & $\mathrm{AF}$ & 10-100 & 11 & -15.7 & 1.6 & & \\
\hline $13 \mathrm{R}-2,59-61$ & 174.36 & 40 & Aphyric amygdaloidal pillow basalt & WWU & $\mathrm{AF}$ & $20-55$ & 10 & -29.1 & 1.3 & -29.2 & 1.3 \\
\hline $14 \mathrm{R}-1,14-16$ & 177.54 & 40 & Aphyric amygdaloidal pillow basalt & Shipboard & $\mathrm{TH}$ & $300-500$ & 7 & -22.3 & 1.6 & & \\
\hline $14 \mathrm{R}-1,136-138$ & 178.76 & 45 & Aphyric amygdaloidal basalt & WWU & $\mathrm{TH}$ & $400-540$ & 7 & -26.5 & 4.0 & -26.2 & 4.3 \\
\hline $14 \mathrm{R}-2,32-34$ & 179.16 & 46 & Aphyric amygdaloidal basalt & Shipboard & $\mathrm{TH}$ & $100-525$ & 11 & -24.1 & 1.7 & & \\
\hline $14 \mathrm{R}-2,121-123$ & 180.05 & 47 & Aphyric amygdaloidal basalt & Shipboard & $\mathrm{TH}$ & $100-475$ & 9 & -24.3 & 2.2 & & \\
\hline $15 R-1,6-9$ & 182.26 & 50 & Aphyric amygdaloidal basalt & Shipboard & $\mathrm{AF}$ & $10-80$ & 10 & -17.2 & 0.7 & & \\
\hline $16 \mathrm{R}-1,78-80$ & 187.78 & 52 & Aphyric amygdaloidal basalt & WWU & $\mathrm{AF}$ & $25-55$ & 7 & -23.1 & 2.9 & -24 & 1.7 \\
\hline $16 \mathrm{R}-2,35-37$ & 188.31 & 54 & Aphyric amygdaloidal basalt & WWU & $\mathrm{TH}$ & $310-450$ & 4 & -20.5 & 0.7 & -20.6 & 0.9 \\
\hline 16R-2, 89-91 & 188.85 & 56 & Aphyric amygdaloidal basalt & Shipboard & $\mathrm{TH}$ & $300-525$ & 9 & -31.8 & 3.4 & & \\
\hline
\end{tabular}

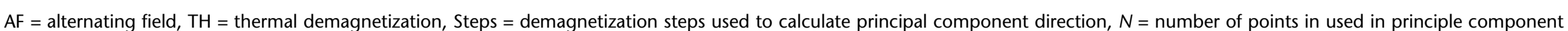

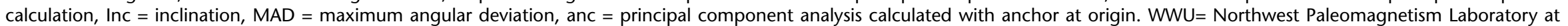
Western Washington University (USA). 
Table T2. Discrete sample paleomagnetic results, Hole U1347A. (Continued on next page.)

\begin{tabular}{|c|c|c|c|c|c|c|c|c|c|c|c|}
\hline $\begin{array}{l}\text { Core, section, } \\
\text { interval }(\mathrm{cm})\end{array}$ & $\begin{array}{l}\text { Depth } \\
\text { (mbsf) }\end{array}$ & $\begin{array}{l}\text { Flow } \\
\text { unit }\end{array}$ & Lithology & $\begin{array}{l}\text { Analysis } \\
\text { location }\end{array}$ & $\begin{array}{c}\text { Demagnetization } \\
\text { treatment }\end{array}$ & $\begin{array}{c}\text { Steps } \\
\left(\mathrm{mT},{ }^{\circ} \mathrm{C}\right)\end{array}$ & $N$ & $\begin{array}{c}\text { Inc anc } \\
\left({ }^{\circ}\right)\end{array}$ & $\begin{array}{l}\text { MAD anc } \\
\left({ }^{\circ}\right)\end{array}$ & $\begin{array}{l}\text { Inc } \\
\left({ }^{\circ}\right)\end{array}$ & $\begin{array}{c}\text { MAD } \\
\left({ }^{\circ}\right)\end{array}$ \\
\hline \multicolumn{12}{|l|}{ 324-U1347A- } \\
\hline $12 \mathrm{R}-1,20.5-22.5$ & 159.90 & 4 & Aphyric basalt & Shipboard & $\mathrm{AF}$ & $12-100$ & 10 & -2.3 & 4.9 & & \\
\hline $12 \mathrm{R}-1,33-35$ & 159.93 & 4 & Aphyric basalt & Shipboard & $\mathrm{TH}$ & $475-600$ & 6 & 18.8 & 12.3 & & \\
\hline 12R-1, 69-71 & 160.29 & 4 & Aphyric basalt & Shipboard & $\mathrm{AF}$ & $12-100$ & 10 & -14.2 & 1.2 & & \\
\hline $12 \mathrm{R}-1,78-80$ & 160.38 & 4 & Aphyric basalt & Shipboard & $\mathrm{AF}$ & $25-100$ & 7 & -1.4 & 3.7 & & \\
\hline 12R-1, 93-95 & 160.53 & 4 & Aphyric basalt & WWU & $\mathrm{TH}$ & $310-520$ & 7 & 54.1 & 4.4 & 56.3 & 3.2 \\
\hline $12 \mathrm{R}-1,104-106$ & 160.64 & 4 & Aphyric basalt & WWU & $\mathrm{AF}$ & $25-60$ & 7 & 4.4 & 2.9 & 4.4 & 2.9 \\
\hline $12 \mathrm{R}-2,2-4$ & 160.96 & 4 & Aphyric basalt & WWU & $\mathrm{AF}$ & $20-65$ & 10 & 4.1 & 3.3 & 3.8 & 2.9 \\
\hline $12 \mathrm{R}-2,9-11$ & 161.03 & 4 & Aphyric basalt & Shipboard & $\mathrm{AF}$ & $10-100$ & 11 & 7.9 & 3.3 & & \\
\hline $12 \mathrm{R}-2,42-44$ & 161.36 & 4 & Aphyric basalt & Shipboard & $\mathrm{TH}$ & $400-600$ & 10 & 34.0 & 4.7 & & \\
\hline $12 \mathrm{R}-2,81-83$ & 161.75 & 4 & Aphyric basalt & WWU & $\mathrm{TH}$ & $480-550$ & 6 & 4.6 & 6.2 & 4.6 & 6.2 \\
\hline $13 \mathrm{R}-1,7-9$ & 167.07 & 4 & Aphyric basalt & WWU & TH & $310-520$ & 7 & 42.0 & 7.5 & 46.6 & 3.6 \\
\hline $13 \mathrm{R}-1,24-26$ & 167.24 & 4 & Aphyric basalt & Shipboard & $\mathrm{TH}$ & $500-600$ & 5 & 26.1 & 5.8 & & \\
\hline $13 R-2,14-16$ & 168.63 & 4 & Aphyric basalt & WWU & $\mathrm{AF}$ & $20-60$ & 8 & 13.7 & 3.5 & 15.5 & 3.4 \\
\hline 13R-2, 90-92 & 169.39 & 4 & Aphyric basalt & Shipboard & TH & $500-600$ & 5 & 50.2 & 5.7 & & \\
\hline $13 R-3,23-25$ & 170.05 & 4 & Aphyric basalt & WWU & TH & $360-520$ & 6 & 18.4 & 6.8 & 18.5 & 9.6 \\
\hline $13 R-4,2-4$ & 170.96 & 4 & Aphyric basalt & Shipboard & $\mathrm{AF}$ & $15-120$ & 11 & 23.0 & 2.5 & & \\
\hline $13 R-6,13-15$ & 172.95 & 4 & Aphyric basalt & Shipboard & $\mathrm{TH}$ & $500-600$ & 5 & 37.5 & 8.8 & & \\
\hline $13 R-6,132-136$ & 174.14 & 4 & Aphyric basalt & WWU & $\mathrm{AF}$ & $40-110$ & 9 & 18.5 & 3.2 & 17.5 & 3.5 \\
\hline $14 R-1,51-53$ & 177.11 & 5 & Amygdaloidal basalt & WWU & $\mathrm{AF}$ & $30-65$ & 8 & 13.3 & 5.1 & 14 & 6.8 \\
\hline $14 \mathrm{R}-1,84-86$ & 177.44 & 5 & Amygdaloidal basalt & WWU & TH & $360-550$ & 9 & 14.3 & 11.2 & 14.5 & 13.6 \\
\hline 14R-1, 145-147 & 178.05 & 5 & Amygdaloidal basalt & Shipboard & TH & $350-600$ & 10 & 23.3 & 11.0 & & \\
\hline 14R-2, 110-112 & 179.17 & 5 & Amygdaloidal basalt & WWU & TH & $310-520$ & 7 & 13.8 & 1.4 & 13.8 & 1.5 \\
\hline $15 R-1,13-15$ & 186.33 & 5 & Amygdaloidal basalt & Shipboard & TH & $500-600$ & 5 & 26.7 & 5.5 & & \\
\hline $15 \mathrm{R}-2,15-17$ & 187.62 & 7 & Amygdaloidal basalt & WWU & $\mathrm{AF}$ & $20-80$ & 10 & 23.7 & 1.2 & 23.6 & 1.3 \\
\hline $16 \mathrm{R}-1,21-23$ & 195.01 & 9 & Amygdaloidal basalt & WWU & TH & $360-520$ & 6 & 37.3 & 4.2 & 35.4 & 5.7 \\
\hline 16R-1, 89-91 & 195.69 & 9 & Amygdaloidal basalt & Shipboard & TH & $500-600$ & 5 & 39.1 & 7.3 & & \\
\hline $16 \mathrm{R}-2,14-16$ & 196.44 & 9 & Amygdaloidal basalt & WWU & $\mathrm{AF}$ & $30-70$ & 7 & 18.6 & 4.2 & 18.3 & 4.8 \\
\hline $16 \mathrm{R}-3,29-31$ & 198.07 & 9 & Amygdaloidal basalt & WWU & TH & $280-450$ & 5 & 64.3 & 6.4 & 68.2 & 4.9 \\
\hline $16 \mathrm{R}-4,10-12$ & 198.37 & 9 & Amygdaloidal basalt & Shipboard & TH & $475-600$ & 6 & 3.2 & 27.1 & & \\
\hline 16R-5, 107-109 & 200.65 & 9 & Amygdaloidal basalt & Shipboard & $\mathrm{AF}$ & $20-100$ & 8 & 19.3 & 1.4 & & \\
\hline $16 \mathrm{R}-5,116-118$ & 200.74 & 9 & Amygdaloidal basalt & WWU & $\mathrm{AF}$ & $25-60$ & 7 & 13.2 & 3.3 & 13.1 & 4.0 \\
\hline $17 \mathrm{R}-2,54-56$ & 206.37 & 11 & Plagioclase phyric basalt & Shipboard & TH & $425-600$ & 8 & 15.6 & 4.5 & & \\
\hline 17R-2, 94-96 & 206.77 & 11 & Plagioclase phyric basalt & WWU & TH & $310-520$ & 7 & 15.1 & 3.0 & 15.4 & 3.4 \\
\hline $17 R-3,86-88$ & 208.15 & 11 & Plagioclase phyric basalt & WWU & $\mathrm{AF}$ & $20-70$ & 9 & 18.0 & 1.4 & 18.1 & 1.4 \\
\hline 17R-3, 107-109 & 208.36 & 11 & Plagioclase phyric basalt & Shipboard & TH & $500-600$ & 5 & 16.0 & 4.7 & & \\
\hline $18 \mathrm{R}-1,44-46$ & 214.44 & 11 & Plagioclase phyric basalt & Shipboard & $\mathrm{AF}$ & $10-100$ & 11 & 10.2 & 4.3 & & \\
\hline $18 R-3,75-77$ & 217.34 & 11 & Plagioclase phyric basalt & Shipboard & $\mathrm{AF}$ & $10-100$ & 11 & 12.9 & 1.1 & & \\
\hline $18 \mathrm{R}-3,143-145$ & 218.01 & 13 & Aphyric amygdaloidal basalt & WWU & $\mathrm{TH}$ & $280-450$ & 5 & 33.0 & 5.4 & 27.3 & 4.6 \\
\hline $18 R-4,54-56$ & 218.58 & 14 & Aphyric amygdaloidal basalt & WWU & $\mathrm{AF}$ & $35-120$ & 11 & -2.0 & 1.4 & -2.1 & 1.5 \\
\hline $18 R-5,66-68$ & 220.12 & 17 & Sparsely plagioclase phyric basalt & WWU & $\mathrm{TH}$ & $280-450$ & 5 & 9.9 & 2.1 & 10.9 & 2.3 \\
\hline $18 \mathrm{R}-5,82-84$ & 220.28 & 17 & Sparsely plagioclase phyric basalt & Shipboard & TH & $400-550$ & 7 & 24.2 & 11.8 & & \\
\hline $18 \mathrm{R}-6,34-36$ & 221.24 & 19 & Sparsely plagioclase phyric basalt & WWU & $\mathrm{AF}$ & $30-100$ & 10 & 12.5 & 1.9 & 12.6 & 2.2 \\
\hline $19 R-1,32-34$ & 223.92 & 20 & Aphyric basalt & Shipboard & $\mathrm{AF}$ & $15-80$ & 8 & 20.8 & 9.0 & & \\
\hline 19R-1, 117-119 & 224.77 & 22 & Aphyric basalt & WWU & TH & $280-450$ & 6 & 10.3 & 2.6 & 10.9 & 4.1 \\
\hline $19 \mathrm{R}-2,100-102$ & 225.88 & 26 & Aphyric basalt & WWU & $\mathrm{AF}$ & $25-120$ & 13 & 4.2 & 1.8 & 4.3 & 1.8 \\
\hline $19 R-3,14-16$ & 226.44 & 26 & Aphyric basalt & WWU & $\mathrm{TH}$ & $280-450$ & 5 & 10.0 & 2.4 & 11.8 & 3.0 \\
\hline $19 R-4,8-10$ & 227.70 & 29 & Aphyric basalt & Shipboard & TH & $375-600$ & 10 & 22.3 & 8.3 & & \\
\hline $19 R-4,20-22$ & 227.82 & 29 & Aphyric basalt & WWU & $\mathrm{AF}$ & $35-100$ & 9 & 0.9 & 1.1 & 1.2 & 1.3 \\
\hline $20 \mathrm{R}-1,22-24$ & 233.42 & 30 & Plagioclase phyric basalt & WWU & $\mathrm{AF}$ & $25-100$ & 11 & 8.7 & 2.5 & 8.8 & 2.7 \\
\hline $20 \mathrm{R}-2,44-46$ & 235.08 & 33 & Plagioclase phyric basalt & WWU & $\mathrm{TH}$ & $280-450$ & 5 & 24.3 & 2.4 & 25.9 & 2.1 \\
\hline $20 \mathrm{R}-3,5-7$ & 235.90 & 33 & Plagioclase phyric basalt & WWU & $\mathrm{AF}$ & $25-100$ & 11 & 5.5 & 2.7 & 5.8 & 3.0 \\
\hline $20 \mathrm{R}-3,46-48$ & 236.31 & 34 & Plagioclase phyric basalt & Shipboard & $\mathrm{AF}$ & $25-100$ & 7 & 3.8 & 3.9 & & \\
\hline $20 \mathrm{R}-3,118-120$ & 237.03 & 34 & Plagioclase phyric basalt & Shipboard & TH & $200-600$ & 14 & 46.3 & 6.1 & & \\
\hline $21 \mathrm{R}-3,72-74$ & 245.21 & 37 & Plagioclase phyric basalt & Shipboard & TH & $300-500$ & 9 & 5.9 & 5.9 & & \\
\hline $21 R-3,14-16$ & 247.39 & 37 & Plagioclase phyric basalt & Shipboard & $\mathrm{AF}$ & $10-100$ & 11 & 26.6 & 1.5 & & \\
\hline $21 \mathrm{R}-4,12-14$ & 246.11 & 37 & Plagioclase phyric basalt & WWU & $\mathrm{TH}$ & $310-450$ & 4 & 25.8 & 3.8 & 33.2 & 2.6 \\
\hline $21 \mathrm{R}-4,121-123$ & 253.61 & 40 & Aphyric basalt & Shipboard & $\mathrm{AF}$ & $12-60$ & 8 & 24.3 & 1.2 & & \\
\hline $21 \mathrm{R}-5,65-67$ & 247.90 & 41 & Aphyric basalt & WWU & $\mathrm{AF}$ & $30-50$ & 5 & 18.8 & 8.5 & 15.3 & 9.2 \\
\hline $22 \mathrm{R}-1,51-53$ & 252.91 & 41 & Aphyric basalt & WWU & TH & $280-450$ & 5 & 35.8 & 3.5 & 37.1 & 4.5 \\
\hline $22 \mathrm{R}-2,74-76$ & 254.60 & 42 & Amygdaloidal basalt & WWU & $\mathrm{AF}$ & $15-45$ & 7 & 14.7 & 1.0 & 14.8 & 1.0 \\
\hline 22R-2, 95-97 & 254.81 & 42 & Amygdaloidal basalt & Shipboard & $\mathrm{TH}$ & $475-600$ & 6 & 17.5 & 12.2 & & \\
\hline $22 \mathrm{R}-3,70-72$ & 255.97 & 42 & Amygdaloidal basalt & Shipboard & TH & $475-600$ & 6 & 25.0 & 17.9 & & \\
\hline 22R-4, 12-14 & 256.84 & 42 & Amygdaloidal basalt & Shipboard & TH & $150-600$ & 10 & 43.8 & 5.6 & & \\
\hline $22 \mathrm{R}-4,135-137$ & 258.06 & 42 & Amygdaloidal basalt & Shipboard & $\mathrm{AF}$ & $10-80$ & 10 & 24.2 & 0.9 & & \\
\hline 22R-5, 9-11 & 258.19 & 42 & Amygdaloidal basalt & WWU & $\mathrm{AF}$ & $25-60$ & 8 & 21.8 & 6.5 & 23.8 & 6.7 \\
\hline $22 \mathrm{R}-5,61-63$ & 258.71 & 44 & Plagioclase phyric basalt & WWU & TH & $500-550$ & 5 & 11.8 & 5.0 & 12.6 & 9.5 \\
\hline $22 \mathrm{R}-5,86-88$ & 258.96 & 46 & Plagioclase phyric basalt & Shipboard & TH & $200-600$ & 13 & 9.7 & 6.2 & & \\
\hline $23 R-2,76-78$ & 262.98 & 48 & Sparsely plagioclase phyric basalt & Shipboard & $\mathrm{TH}$ & $425-600$ & 8 & 15.9 & 7.0 & & \\
\hline
\end{tabular}


Table T2 (continued).

\begin{tabular}{|c|c|c|c|c|c|c|c|c|c|c|c|}
\hline $\begin{array}{l}\text { Core, section, } \\
\text { interval }(\mathrm{cm})\end{array}$ & $\begin{array}{l}\text { Depth } \\
\text { (mbsf) }\end{array}$ & $\begin{array}{l}\text { Flow } \\
\text { unit }\end{array}$ & Lithology & $\begin{array}{l}\text { Analysis } \\
\text { location }\end{array}$ & $\begin{array}{l}\text { Demagnetization } \\
\text { treatment }\end{array}$ & $\begin{array}{c}\text { Steps } \\
\left(\mathrm{mT},{ }^{\circ} \mathrm{C}\right)\end{array}$ & $N$ & $\begin{array}{c}\text { Inc anc } \\
\left({ }^{\circ}\right)\end{array}$ & $\begin{array}{l}\text { MAD anc } \\
\left({ }^{\circ}\right)\end{array}$ & $\begin{array}{c}\operatorname{Inc} \\
\left({ }^{\circ}\right)\end{array}$ & $\begin{array}{l}\text { MAD } \\
\left({ }^{\circ}\right)\end{array}$ \\
\hline $23 \mathrm{R}-2,134-136$ & 263.56 & 48 & Sparsely plagioclase phyric basalt & WWU & TH & $220-450$ & 5 & 15.2 & 2.6 & 15.9 & 2.8 \\
\hline $23 \mathrm{R}-3,9-11$ & 263.72 & 49 & Sparsely plagioclase phyric basalt & Shipboard & TH & $200-600$ & 13 & 29.3 & 3.0 & & \\
\hline $23 R-3,34-36$ & 265.38 & 49 & Sparsely plagioclase phyric basalt & Shipboard & $\mathrm{AF}$ & $20-120$ & 9 & 13.0 & 3.5 & & \\
\hline $23 R-3,62-64$ & 264.26 & 50 & Sparsely plagioclase phyric basalt & WWU & $\mathrm{AF}$ & $30-80$ & 11 & 3.8 & 1.7 & 3.9 & 1.7 \\
\hline $23 R-4,103-105$ & 266.07 & 53 & Sparsely plagioclase phyric basalt & WWU & TH & $280-450$ & 5 & 10.7 & 3.4 & 13.2 & 5.1 \\
\hline $23 R-6,96-98$ & 267.79 & 53 & Sparsely plagioclase phyric basalt & Shipboard & TH & $450-600$ & 7 & 18.7 & 5.7 & & \\
\hline $24 \mathrm{R}-1,34-36$ & 271.94 & 53 & Sparsely plagioclase phyric basalt & Shipboard & TH & $350-600$ & 11 & 39.7 & 19.0 & & \\
\hline $24 R-3,61-63$ & 274.70 & 53 & Sparsely plagioclase phyric basalt & Shipboard & $\mathrm{AF}$ & $10-100$ & 11 & 8.0 & 1.6 & & \\
\hline $24 \mathrm{R}-3,136-138$ & 275.45 & 56 & Sparsely plagioclase phyric basalt & WWU & $\mathrm{AF}$ & $40-75$ & 8 & 0.8 & 2.4 & 0.7 & 3.0 \\
\hline $24 R-5,41-43$ & 277.38 & 57 & Sparsely plagioclase phyric basalt & Shipboard & $\mathrm{AF}$ & $20-140$ & 9 & 16.4 & 2.5 & & \\
\hline $24 R-5,49-51$ & 277.46 & 57 & Sparsely plagioclase phyric basalt & WWU & TH & $280-450$ & 5 & 21.7 & 6.6 & 20.6 & 6.8 \\
\hline $24 \mathrm{R}-6,30-32$ & 278.45 & 60 & Sparsely plagioclase phyric basalt & Shipboard & $\mathrm{AF}$ & $15-100$ & 9 & 13.6 & 1.7 & & \\
\hline 24R-6, 69-71 & 278.84 & 60 & Sparsely plagioclase phyric basalt & WWU & $\mathrm{AF}$ & $30-55$ & 6 & 15.4 & 7.8 & 18.4 & 7.0 \\
\hline $24 \mathrm{R}-6,90-92$ & 279.05 & 60 & Sparsely plagioclase phyric basalt & Shipboard & TH & $200-600$ & 10 & 32.3 & 6.4 & & \\
\hline $24 \mathrm{R}-7,45-47$ & 279.81 & 61 & Sparsely plagioclase phyric basalt & Shipboard & TH & $450-600$ & 7 & -7.1 & 0.7 & & \\
\hline $24 \mathrm{R}-8,24-26$ & 280.64 & 62 & Sparsely plagioclase phyric basalt & Shipboard & $\mathrm{TH}$ & $400-600$ & 9 & 30.2 & 5.2 & & \\
\hline $24 R-8,65-67$ & 281.05 & 64 & Sparsely plagioclase phyric basalt & WWU & TH & $360-550$ & 9 & 13.5 & 14.9 & 14.3 & 16.2 \\
\hline $24 \mathrm{R}-8,127-129$ & 281.67 & 65 & Sparsely plagioclase phyric basalt & WWU & $\mathrm{AF}$ & $25-60$ & 8 & 7.7 & 2.7 & 7.8 & 3.0 \\
\hline $25 \mathrm{R}-1,44-46$ & 281.64 & 65 & Sparsely plagioclase phyric basalt & Shipboard & $\mathrm{AF}$ & $15-100$ & 9 & 13.9 & 2.9 & & \\
\hline $25 \mathrm{R}-1,84-86$ & 282.04 & 65 & Sparsely plagioclase phyric basalt & WWU & $\mathrm{AF}$ & $45-80$ & 8 & 1.5 & 5.3 & 2.2 & 7.4 \\
\hline $25 \mathrm{R}-2,81-83$ & 283.31 & 67 & Sparsely plagioclase phyric basalt & Shipboard & TH & $350-575$ & 10 & 43.2 & 8.6 & & \\
\hline $25 R-3,65-67$ & 284.53 & 68 & Sparsely plagioclase phyric basalt & Shipboard & $\mathrm{AF}$ & $20-80$ & 7 & 6.2 & 4.3 & & \\
\hline $25 R-4,102-104$ & 286.40 & 69 & Sparsely plagioclase phyric basalt & Shipboard & $\mathrm{TH}$ & $450-600$ & 9 & 35.3 & 3.9 & & \\
\hline $25 R-4,125-127$ & 286.63 & 70 & Sparsely plagioclase phyric basalt & WWU & $\mathrm{AF}$ & $25-75$ & 11 & 5.2 & 1.3 & 5.3 & 1.2 \\
\hline $25 \mathrm{R}-5,59-61$ & 287.34 & 71 & Sparsely plagioclase phyric basalt & Shipboard & TH & $300-600$ & 13 & 48.9 & 4.6 & & \\
\hline $26 \mathrm{R}-1,58-60$ & 291.28 & 79 & Sparsely plagioclase phyric basalt & Shipboard & TH & $475-600$ & 6 & -10.4 & 12.8 & & \\
\hline 26R-1, 103-105 & 291.73 & 79 & Sparsely plagioclase phyric basalt & WWU & TH & $310-450$ & 4 & 8.0 & 2.3 & 9.3 & 3.3 \\
\hline $26 \mathrm{R}-2,29-31$ & 292.49 & 81 & Sparsely plagioclase phyric basalt & WWU & $\mathrm{AF}$ & $25-65$ & 9 & -4.0 & 2.9 & -2.6 & 3.1 \\
\hline $26 \mathrm{R}-2,84-86$ & 293.04 & 81 & Sparsely plagioclase phyric basalt & Shipboard & TH & $150-350$ & 6 & 80.4 & 4.6 & & \\
\hline $27 \mathrm{R}-2,23-25$ & 294.74 & 81 & Sparsely plagioclase phyric basalt & Shipboard & $\mathrm{AF}$ & $20-80$ & 7 & 14.1 & 5.1 & & \\
\hline $27 R-2,78-80$ & 295.29 & 81 & Sparsely plagioclase phyric basalt & WWU & $\mathrm{AF}$ & $20-65$ & 10 & 10.3 & 4.2 & 7.1 & 3.4 \\
\hline $27 \mathrm{R}-3,8-10$ & 295.69 & 81 & Sparsely plagioclase phyric basalt & WWU & TH & $360-450$ & 3 & 1.4 & 7.9 & 43.5 & 26.3 \\
\hline $27 R-3,65-67$ & 296.24 & 81 & Sparsely plagioclase phyric basalt & WWU & $\mathrm{AF}$ & $30-50$ & 5 & -7.9 & 3.4 & -3.2 & 14.2 \\
\hline $27 R-4,63-65$ & 297.10 & 81 & Sparsely plagioclase phyric basalt & Shipboard & TH & $450-600$ & 9 & -38.7 & 44.8 & & \\
\hline $27 R-5,4-6$ & 297.90 & 81 & Sparsely plagioclase phyric basalt & WWU & $\mathrm{AF}$ & $25-65$ & 9 & -1.7 & 2.8 & -1.0 & 3.2 \\
\hline $27 \mathrm{R}-5,120-122$ & 299.06 & 81 & Sparsely plagioclase phyric basalt & WWU & TH & $120-310$ & 5 & 88.5 & 1.6 & 88.3 & 1.5 \\
\hline $27 \mathrm{R}-6,22-24$ & 299.37 & 81 & Sparsely plagioclase phyric basalt & WWU & $\mathrm{AF}$ & $15-45$ & 7 & 21.4 & 10.6 & 24.1 & 11.0 \\
\hline $27 \mathrm{R}-6,123-125$ & 300.38 & 81 & Sparsely plagioclase phyric basalt & Shipboard & TH & $150-325$ & 5 & 55.6 & 7.2 & & \\
\hline $28 \mathrm{R}-1,8-10$ & 300.48 & 81 & Sparsely plagioclase phyric basalt & WWU & TH & $220-450$ & 6 & 80.3 & 2.2 & 81.0 & 1.4 \\
\hline $28 \mathrm{R}-1,67-69$ & 301.07 & 81 & Sparsely plagioclase phyric basalt & Shipboard & $\mathrm{AF}$ & $12-60$ & 8 & 79.3 & 4.5 & & \\
\hline $28 \mathrm{R}-2,28-30$ & 301.92 & 81 & Sparsely plagioclase phyric basalt & WWU & $\mathrm{AF}$ & $20-45$ & 6 & 19.9 & 7.1 & 20.9 & 7.7 \\
\hline $28 \mathrm{R}-3,44-46$ & 303.39 & 81 & Sparsely plagioclase phyric basalt & Shipboard & TH & $450-550$ & 7 & -6.9 & 14.1 & & \\
\hline $28 R-5,16-18$ & 305.61 & 81 & Sparsely plagioclase phyric basalt & Shipboard & AF & $20-50$ & 5 & 26.5 & 9.5 & & \\
\hline $28 \mathrm{R}-6,49-51$ & 307.15 & 81 & Sparsely plagioclase phyric basalt & Shipboard & TH & $425-600$ & 8 & -75.7 & 22.6 & & \\
\hline $28 R-7,13-15$ & 308.11 & 81 & Sparsely plagioclase phyric basalt & WWU & TH & $180-310$ & 6 & 82.1 & 1.9 & 82.2 & 1.7 \\
\hline $28 \mathrm{R}-8,80-82$ & 310.04 & 81 & Sparsely plagioclase phyric basalt & Shipboard & $\mathrm{AF}$ & $7-60$ & 10 & 77.8 & 10.2 & & \\
\hline $29 \mathrm{R}-1,23-25$ & 310.13 & 81 & Sparsely plagioclase phyric basalt & Shipboard & TH & $350-600$ & 11 & 25.4 & 25.1 & & \\
\hline $29 \mathrm{R}-3,68-70$ & 312.89 & 81 & Sparsely plagioclase phyric basalt & WWU & $\mathrm{AF}$ & $15-40$ & 6 & 64.2 & 4.6 & 65.4 & 3.5 \\
\hline $29 \mathrm{R}-4,57-59$ & 314.22 & 81 & Sparsely plagioclase phyric basalt & Shipboard & TH & $150-325$ & 5 & 63.4 & 4.1 & & \\
\hline $29 R-4,142-144$ & 315.07 & 82 & Sparsely plagioclase phyric basalt & Shipboard & TH & $350-600$ & 11 & 30.7 & 2.6 & & \\
\hline $29 \mathrm{R}-5,86-88$ & 316.01 & 82 & Sparsely plagioclase phyric basalt & WWU & $\mathrm{AF}$ & $35-55$ & 5 & 9.3 & 7.4 & 5.3 & 10.3 \\
\hline
\end{tabular}

$\mathrm{AF}=$ alternating field, $\mathrm{TH}=$ thermal demagnetization, $\mathrm{Steps}=$ demagnetization steps used to calculate principal component direction, $\mathrm{N}=$ number of points in used in principle component calculation, Inc = inclination, MAD = maximum angular deviation, anc = principal component analysis calculated with anchor at origin. WWU $=$ Northwest Paleomagnetism Laboratory at Western Washington University (USA). 
Table T3. Discrete sample paleomagnetic results, Hole U1350A. (Continued on next page.)

\begin{tabular}{|c|c|c|c|c|c|c|c|c|c|c|c|}
\hline $\begin{array}{l}\text { Core, section, } \\
\text { interval }(\mathrm{cm})\end{array}$ & $\begin{array}{l}\text { Depth } \\
\text { (mbsf) }\end{array}$ & $\begin{array}{l}\text { Flow } \\
\text { unit }\end{array}$ & Lithology & $\begin{array}{l}\text { Analysis } \\
\text { location }\end{array}$ & $\begin{array}{c}\text { Demagnetization } \\
\text { treatment }\end{array}$ & $\begin{array}{c}\text { Steps } \\
\left(\mathrm{mT},{ }^{\circ} \mathrm{C}\right)\end{array}$ & $N$ & $\begin{array}{c}\text { Inc anc } \\
\left({ }^{\circ}\right)\end{array}$ & $\begin{array}{c}\text { MAD anc } \\
\left({ }^{\circ}\right)\end{array}$ & $\begin{array}{c}\text { Inc } \\
\left({ }^{\circ}\right)\end{array}$ & $\begin{array}{c}\mathrm{MAD} \\
\left({ }^{\circ}\right)\end{array}$ \\
\hline \multicolumn{12}{|l|}{ 324-U1350A- } \\
\hline $7 \mathrm{R}-1,53-55$ & 153.13 & 2 & Aphyric basalt & Shipboard & $\mathrm{TH}$ & $150-550$ & 14 & -9.5 & 2.6 & & \\
\hline 7R-1, 104-106 & 153.64 & 2 & Aphyric basalt & WWU & $\mathrm{TH}$ & $280-530$ & 7 & -1.1 & 2.8 & -0.5 & 3.0 \\
\hline $7 \mathrm{R}-2,21-23$ & 154.25 & 2 & Aphyric basalt & WWU & $\mathrm{AF}$ & $15-40$ & 6 & 5.8 & 3.0 & 6.3 & 3.1 \\
\hline $8 \mathrm{R}-1,18-20$ & 155.48 & 4 & Aphyric basalt & Shipboard & $\mathrm{TH}$ & $325-550$ & 10 & -30.9 & 9.9 & & \\
\hline $8 R-1,63-65$ & 155.93 & 4 & Aphyric basalt & WWU & $\mathrm{TH}$ & $400-530$ & 5 & -2.0 & 2.8 & -3.3 & 3.4 \\
\hline $8 R-2,4-6$ & 156.8 & 6 & Massive basalt & WWU & $\mathrm{AF}$ & $25-55$ & 7 & -6.7 & 1.7 & -6.5 & 1.8 \\
\hline $8 R-3,4-6$ & 158.11 & 7 & Massive basalt & WWU & $\mathrm{AF}$ & $25-45$ & 5 & 9.1 & 1.9 & 9.0 & 2.4 \\
\hline $8 R-3,85-87$ & 158.92 & 7 & Massive basalt & Shipboard & $\mathrm{AF}$ & $7-100$ & 12 & -1.3 & 1.6 & & \\
\hline $9 R-1,58-60$ & 162.88 & 9 & Aphyric vesicular basalt & WWU & $\mathrm{TH}$ & $220-500$ & 8 & -8.0 & 2.8 & -7.8 & 3.1 \\
\hline $9 \mathrm{R}-2,69-71$ & 164.04 & 9 & Aphyric vesicular basalt & Shipboard & $\mathrm{TH}$ & $150-550$ & 14 & 10.0 & 6.3 & & \\
\hline $9 R-3,51-53$ & 164.93 & 9 & Aphyric vesicular basalt & WWU & $\mathrm{AF}$ & $15-60$ & 10 & -4.4 & 1.6 & -4.4 & 1.7 \\
\hline $9 R-4,89-91$ & 166.62 & 10 & Aphyric massive basalt & Shipboard & $\mathrm{AF}$ & $10-100$ & 11 & -2.8 & 0.9 & & \\
\hline 9R-5, 9-11 & 167.07 & 10 & Aphyric massive basalt & WWU & $\mathrm{TH}$ & $220-500$ & 8 & -0.3 & 3.3 & 0.0 & 3.8 \\
\hline $9 R-6,4-6$ & 168.33 & 10 & Aphyric massive basalt & Shipboard & $\mathrm{TH}$ & $100-500$ & 13 & 31.2 & 9.3 & & \\
\hline $9 R-6,67-69$ & 168.96 & 10 & Aphyric massive basalt & WWU & $\mathrm{AF}$ & $20-60$ & 9 & 1.0 & 7.3 & 0.5 & 8.3 \\
\hline $10 \mathrm{R}-1,21-23$ & 172.11 & 11 & Aphyric massive basalt & WWU & $\mathrm{TH}$ & $280-440$ & 5 & 35.2 & 8.9 & 39.9 & 4.2 \\
\hline $10 \mathrm{R}-2,38-40$ & 173.73 & 12 & Aphyric massive basalt & WWU & $\mathrm{AF}$ & $15-50$ & 8 & 2.5 & 1.0 & 2.5 & 1.0 \\
\hline $11 \mathrm{R}-1,51-53$ & 182.01 & 12 & Aphyric massive basalt & Shipboard & $\mathrm{AF}$ & $10-80$ & 10 & 0.5 & 0.6 & & \\
\hline $11 \mathrm{R}-1,128-130$ & 182.78 & 13 & Aphyric massive basalt & WWU & $\mathrm{TH}$ & $440-530$ & 4 & -3.6 & 1.8 & -4.7 & 5.2 \\
\hline $11 \mathrm{R}-2,14-16$ & 183.16 & 14 & Aphyric massive basalt & WWU & $\mathrm{AF}$ & $20-45$ & 6 & -2.4 & 2.6 & -2.8 & 2.7 \\
\hline $11 \mathrm{R}-2,77-79$ & 183.79 & 14 & Aphyric massive basalt & Shipboard & $\mathrm{TH}$ & $150-550$ & 14 & 7.6 & 6.2 & & \\
\hline $12 \mathrm{R}-1,8-10$ & 191.18 & 14 & Aphyric massive basalt & WWU & $\mathrm{AF}$ & $25-50$ & 6 & 18.3 & 2.7 & 17.5 & 2.6 \\
\hline $12 \mathrm{R}-1,50-52$ & 191.6 & 15 & Plagioclase phyric massive basalt & Shipboard & $\mathrm{AF}$ & $10-80$ & 11 & 27.0 & 1.1 & & \\
\hline $13 \mathrm{R}-1,50-52$ & 196.4 & 16 & Plagioclase phyric massive basalt & Shipboard & $\mathrm{TH}$ & $150-550$ & 14 & 43.0 & 4.9 & & \\
\hline $13 \mathrm{R}-1,100-102$ & 196.9 & 16 & Plagioclase phyric massive basalt & WWU & $\mathrm{TH}$ & $310-500$ & 6 & -19.7 & 5.2 & -19.1 & 5.6 \\
\hline $13 R-2,55-57$ & 197.88 & 17 & Plagioclase phyric massive basalt & WWU & $\mathrm{AF}$ & $30-50$ & 5 & 7.3 & 3.9 & 7.3 & 4.7 \\
\hline $14 R-1,52-54$ & 201.22 & 20 & Plagioclase phyric massive basalt & WWU & $\mathrm{AF}$ & $35-65$ & 7 & 2.5 & 2.0 & 2.0 & 1.8 \\
\hline $14 \mathrm{R}-1,78-80$ & 201.48 & 20 & Plagioclase phyric massive basalt & Shipboard & $\mathrm{TH}$ & $300-550$ & 9 & 27.3 & 9.5 & & \\
\hline $14 \mathrm{R}-2,9-11$ & 201.96 & 20 & Plagioclase phyric massive basalt & Shipboard & $\mathrm{AF}$ & $10-80$ & 10 & -1.0 & 1.3 & & \\
\hline $14 \mathrm{R}-2,21-23$ & 202.08 & 20 & Plagioclase phyric massive basalt & WWU & $\mathrm{TH}$ & $440-550$ & 7 & -1.2 & 1.0 & -1.0 & 1.1 \\
\hline $15 \mathrm{R}-1,53-55$ & 206.03 & 25 & Plagioclase phyric massive basalt & WWU & $\mathrm{AF}$ & $25-50$ & 6 & -3.3 & 2.7 & -4.1 & 2.7 \\
\hline $15 R-1,101-103$ & 206.51 & 25 & Plagioclase phyric massive basalt & WWU & $\mathrm{TH}$ & $280-440$ & 5 & 38.1 & 5.3 & 37.8 & 6.1 \\
\hline 16R-1, 35-37 & 210.65 & 27 & Plagioclase phyric massive basalt & Shipboard & TH & $300-575$ & 12 & -2.3 & 2.0 & & \\
\hline $16 \mathrm{R}-2,5-7$ & 211.07 & 27 & Plagioclase phyric massive basalt & WWU & $\mathrm{TH}$ & $310-530$ & 7 & -6.0 & 1.9 & -5.3 & 2.2 \\
\hline $16 \mathrm{R}-2,72-74$ & 211.74 & 27 & Plagioclase phyric massive basalt & Shipboard & $\mathrm{AF}$ & $15-100$ & 10 & -3.5 & 1.8 & & \\
\hline $16 \mathrm{R}-3,105-107$ & 213.01 & 28 & Aphyric massive basalt & WWU & $\mathrm{AF}$ & $25-55$ & 7 & -2.5 & 1.7 & -2.4 & 1.9 \\
\hline $17 \mathrm{R}-1,138-140$ & 221.28 & 30 & Phyric massive basalt & WWU & $\mathrm{TH}$ & $360-530$ & 6 & 10.2 & 1.3 & 10.6 & 1.5 \\
\hline $17 \mathrm{R}-2,34-36$ & 221.74 & 30 & Phyric massive basalt & Shipboard & $\mathrm{TH}$ & $150-575$ & 15 & 18.3 & 3.3 & & \\
\hline $17 \mathrm{R}-2,80-82$ & 222.2 & 30 & Phyric massive basalt & WWU & $\mathrm{AF}$ & $25-45$ & 5 & 0.4 & 1.7 & 0.5 & 2.0 \\
\hline $17 \mathrm{R}-3,72-74$ & 223.52 & 34 & Phyric massive basalt & Shipboard & $\mathrm{AF}$ & $10-80$ & 11 & 3.0 & 0.7 & & \\
\hline $18 \mathrm{R}-1,72-74$ & 230.22 & 36 & Aphyric massive basalt & WWU & $\mathrm{TH}$ & $280-440$ & 5 & 24.9 & 4.1 & 23.7 & 4.2 \\
\hline $18 \mathrm{R}-1,97-99$ & 230.47 & 36 & Aphyric massive basalt & Shipboard & $\mathrm{TH}$ & $150-575$ & 51 & 14.8 & 3.4 & & \\
\hline $18 \mathrm{R}-2,50-52$ & 231.44 & 37 & Aphyric vesicular basalt & Shipboard & $\mathrm{AF}$ & $7-60$ & 11 & 14.5 & 0.9 & & \\
\hline $18 R-2,73-75$ & 231.67 & 37 & Aphyric vesicular basalt & WWU & $\mathrm{AF}$ & $30-55$ & 6 & 11.0 & 2.2 & 10.9 & 2.2 \\
\hline $18 \mathrm{R}-3,12-14$ & 232.56 & 37 & Aphyric vesicular basalt & WWU & $\mathrm{TH}$ & $400-500$ & 4 & 46.6 & 4.6 & 60.6 & 8.0 \\
\hline $18 \mathrm{R}-3,60-62$ & 233.04 & 37 & Aphyric vesicular basalt & Shipboard & $\mathrm{TH}$ & $200-575$ & 14 & 29.0 & 1.9 & & \\
\hline 19R-1, 68-70 & 239.78 & 38 & Aphyric massive basalt & Shipboard & $\mathrm{AF}$ & $10-60$ & 10 & 6.3 & 0.7 & & \\
\hline $19 \mathrm{R}-1,119-121$ & 240.29 & 39 & Aphyric massive basalt & WWU & $\mathrm{AF}$ & $30-55$ & 6 & 6.3 & 2.8 & 6.5 & 3.4 \\
\hline $19 \mathrm{R}-2,125-127$ & 241.78 & 44 & Aphyric massive basalt & WWU & $\mathrm{TH}$ & $280-480$ & 6 & 16.0 & 3.4 & 16.0 & 3.8 \\
\hline $19 \mathrm{R}-3,37-39$ & 242.33 & 44 & Aphyric massive basalt & WWU & $\mathrm{AF}$ & $25-50$ & 6 & -8.1 & 2.4 & -8.7 & 2.4 \\
\hline $20 \mathrm{R}-1,31-33$ & 249.01 & 46 & Aphyric massive basalt & Shipboard & $\mathrm{TH}$ & $350-575$ & 10 & 13.4 & 4.5 & & \\
\hline $20 \mathrm{R}-1,65-67$ & 249.35 & 46 & Aphyric massive basalt & WWU & $\mathrm{AF}$ & $25-50$ & 6 & -8.0 & 2.0 & -8.0 & 2.3 \\
\hline 20R-1, 95-97 & 249.65 & 47 & Aphyric massive basalt & WWU & $\mathrm{TH}$ & $480-550$ & 6 & -11.8 & 6.3 & -11.1 & 8.7 \\
\hline $20 \mathrm{R}-2,67-69$ & 250.7 & 49 & Aphyric massive basalt & WWU & TH & $440-575$ & 6 & -5.6 & 3.3 & -5.7 & 4.2 \\
\hline $20 \mathrm{R}-2,132-134$ & 251.35 & 50 & Aphyric massive basalt & WWU & $\mathrm{AF}$ & $25-50$ & 6 & -6.3 & 2.5 & -6.6 & 2.9 \\
\hline $20 \mathrm{R}-3,4-6$ & 251.44 & 50 & Aphyric massive basalt & Shipboard & $\mathrm{AF}$ & $7-60$ & 11 & -11.0 & 1.4 & & \\
\hline $21 \mathrm{R}-1,23-25$ & 258.53 & 51 & Aphyric massive basalt & WWU & $\mathrm{TH}$ & $360-550$ & 8 & 1.6 & 4.0 & 0.6 & 4.6 \\
\hline $21 \mathrm{R}-1,35-37$ & 258.65 & 51 & Aphyric massive basalt & Shipboard & $\mathrm{TH}$ & $325-575$ & 11 & 25.1 & 3.0 & & \\
\hline $21 \mathrm{R}-1,137-139$ & 259.67 & 52 & Aphyric massive basalt & WWU & $\mathrm{AF}$ & $30-50$ & 5 & 5.0 & 1.1 & 4.9 & 1.3 \\
\hline $21 \mathrm{R}-2,67-69$ & 260.47 & 52 & Aphyric massive basalt & Shipboard & $\mathrm{AF}$ & $7-80$ & 12 & 7.9 & 1.1 & & \\
\hline $21 \mathrm{R}-2,135-137$ & 261.15 & 53 & Phyric massive basalt & WWU & $\mathrm{TH}$ & $440-575$ & 6 & 16.7 & 1.2 & 16.7 & 1.4 \\
\hline $21 \mathrm{R}-3,48-50$ & 261.71 & 53 & Phyric massive basalt & Shipboard & $\mathrm{TH}$ & $250-575$ & 13 & 18.6 & 3.5 & & \\
\hline $21 \mathrm{R}-3,64-66$ & 261.87 & 54 & Phyric massive basalt & WWU & $\mathrm{AF}$ & $25-45$ & 5 & 14.1 & 1.6 & 14.2 & 1.9 \\
\hline $22 \mathrm{R}-1,67-69$ & 268.47 & 57 & Aphyric massive basalt & WWU & $\mathrm{TH}$ & $360-550$ & 7 & 3.5 & 5.9 & 2.6 & 7.3 \\
\hline $22 \mathrm{R}-2,68-70$ & 269.54 & 59 & Phyric massive basalt & WWU & $\mathrm{TH}$ & $360-550$ & 7 & 1.9 & 3.2 & 1.9 & 3.7 \\
\hline 22R-2, 97-99 & 269.84 & 59 & Phyric massive basalt & Shipboard & $\mathrm{AF}$ & $12-100$ & 11 & -0.3 & 1.9 & & \\
\hline $22 \mathrm{R}-3,113-115$ & 271.3 & 62 & Phyric massive basalt & Shipboard & $\mathrm{TH}$ & $150-525$ & 13 & 22.8 & 3.3 & & \\
\hline $22 \mathrm{R}-4,87-89$ & 272.43 & 65 & Phyric massive basalt & WWU & $\mathrm{TH}$ & $360-520$ & 6 & 4.1 & 1.6 & 4.3 & 1.9 \\
\hline
\end{tabular}


Table T3 (continued).

\begin{tabular}{|c|c|c|c|c|c|c|c|c|c|c|c|}
\hline $\begin{array}{l}\text { Core, section, } \\
\text { interval }(\mathrm{cm})\end{array}$ & $\begin{array}{l}\text { Depth } \\
\text { (mbsf) }\end{array}$ & $\begin{array}{l}\text { Flow } \\
\text { unit }\end{array}$ & Lithology & $\begin{array}{l}\text { Analysis } \\
\text { location }\end{array}$ & $\begin{array}{l}\text { Demagnetization } \\
\text { treatment }\end{array}$ & $\begin{array}{c}\text { Steps } \\
\left(\mathrm{mT},{ }^{\circ} \mathrm{C}\right)\end{array}$ & $N$ & $\begin{array}{c}\text { Inc anc } \\
\left({ }^{\circ}\right)\end{array}$ & $\begin{array}{c}\text { MAD anc } \\
\left({ }^{\circ}\right)\end{array}$ & $\begin{array}{l}\text { Inc } \\
\left({ }^{\circ}\right)\end{array}$ & $\begin{array}{l}\text { MAD } \\
\left({ }^{\circ}\right)\end{array}$ \\
\hline 22R-5, 71-73 & 273.69 & 69 & Phyric massive basalt & WWU & $\mathrm{AF}$ & $15-55$ & 9 & -0.5 & 1.6 & -0.5 & 1.7 \\
\hline $22 \mathrm{R}-5,135-137$ & 274.33 & 70 & Phyric massive basalt & WWU & $\mathrm{TH}$ & $360-540$ & 8 & -11.7 & 1.0 & -11.8 & 1.2 \\
\hline $23 \mathrm{R}-1,22-24$ & 277.62 & 71 & Phyric massive basalt & WWU & $\mathrm{AF}$ & $25-60$ & 8 & 2.4 & 2.8 & 2.0 & 3.3 \\
\hline $23 \mathrm{R}-1,78-80$ & 278.18 & 72 & Phyric massive basalt & Shipboard & $\mathrm{AF}$ & $15-80$ & 9 & 0.4 & 2.1 & & \\
\hline $23 R-2,49-51$ & 279.31 & 74 & Phyric massive basalt & Shipboard & $\mathrm{TH}$ & $425-575$ & 7 & 3.6 & 3.8 & & \\
\hline $23 \mathrm{R}-3,125-127$ & 281.43 & 78 & Phyric massive basalt & WWU & $\mathrm{AF}$ & $25-50$ & 6 & -5.1 & 1.7 & -4.9 & 1.9 \\
\hline $23 R-4,16-18$ & 281.84 & 79 & Phyric massive basalt & WWU & $\mathrm{TH}$ & $360-520$ & 6 & -9.3 & 1.4 & -9.3 & 1.7 \\
\hline $23 R-4,92-94$ & 282.60 & 81 & Phyric massive basalt & Shipboard & $\mathrm{AF}$ & $10-100$ & 12 & -2.5 & 3.3 & & \\
\hline $23 R-5,6-8$ & 283.13 & 81 & Phyric massive basalt & WWU & $\mathrm{AF}$ & $40-65$ & 6 & -3.6 & 3.4 & -4.4 & 2.1 \\
\hline $24 \mathrm{R}-1,42-44$ & 287.42 & 84 & Phyric massive basalt & WWU & $\mathrm{TH}$ & $400-520$ & 5 & -10.0 & 1.2 & -10.5 & 1.2 \\
\hline 24R-1, 109-111 & 288.09 & 85 & Phyric massive basalt & Shipboard & $\mathrm{TH}$ & $150-550$ & 14 & -4.1 & 1.7 & & \\
\hline $24 \mathrm{R}-2,137-139$ & 289.85 & 89 & Phyric massive basalt & WWU & $\mathrm{AF}$ & $30-50$ & 5 & -7.5 & 1.9 & -7.7 & 1.7 \\
\hline $24 R-3,31-33$ & 290.18 & 90 & Phyric massive basalt & Shipboard & $\mathrm{AF}$ & $12-80$ & 10 & 3.8 & 1.0 & & \\
\hline $25 R-1,24-26$ & 296.94 & 92 & Phyric massive basalt & Shipboard & $\mathrm{TH}$ & $325-550$ & 10 & -1.2 & 2.1 & & \\
\hline $25 \mathrm{R}-1,102-104$ & 297.72 & 94 & Phyric massive basalt & WWU & $\mathrm{AF}$ & $25-50$ & 6 & -3.5 & 3.1 & -3.2 & 3.4 \\
\hline $25 \mathrm{R}-1,112-114$ & 297.82 & 94 & Phyric massive basalt & WWU & $\mathrm{TH}$ & $440-540$ & 6 & -14.2 & 3.6 & -14.8 & 4.1 \\
\hline $25 \mathrm{R}-2,60-62$ & 298.80 & 100 & Phyric massive basalt & WWU & $\mathrm{AF}$ & $25-45$ & 5 & -6.3 & 1.6 & -6.7 & 1.1 \\
\hline $25 R-2,119-121$ & 299.39 & 101 & Phyric massive basalt & Shipboard & $\mathrm{AF}$ & $12-80$ & 10 & -12.0 & 1.1 & & \\
\hline $25 \mathrm{R}-3,83-85$ & 300.53 & 107 & Phyric massive basalt & WWU & $\mathrm{TH}$ & $360-520$ & 6 & -0.4 & 1.5 & 0.3 & 1.4 \\
\hline $25 R-4,71-73$ & 301.75 & 114 & Phyric massive basalt & Shipboard & $\mathrm{TH}$ & $150-550$ & 14 & 4.9 & 2.6 & & \\
\hline $25 R-5,57-59$ & 302.88 & 119 & Phyric massive basalt & Shipboard & $\mathrm{AF}$ & $10-80$ & 11 & -7.5 & 0.8 & & \\
\hline $25 R-5,92-93$ & 303.24 & 120 & Phyric massive basalt & WWU & $\mathrm{AF}$ & $15-45$ & 7 & -3.1 & 1.8 & -3.0 & 1.8 \\
\hline $25 R-6,31-33$ & 303.95 & 124 & Phyric massive basalt & WWU & $\mathrm{TH}$ & $360-520$ & 6 & 1.1 & 1.4 & 1.3 & 1.2 \\
\hline 25R-7, 13-15 & 304.92 & 130 & Plagioclase phyric massive basalt & Shipboard & $\mathrm{TH}$ & $150-550$ & 14 & -1.0 & 1.6 & & \\
\hline 25R-7, 91-93 & 305.69 & 133 & Plagioclase phyric massive basalt & WWU & $\mathrm{AF}$ & $20-45$ & 6 & -1.8 & 1.3 & -1.8 & 1.5 \\
\hline $25 R-8,13-15$ & 306.31 & 138 & Phyric massive basalt & WWU & $\mathrm{TH}$ & $400-520$ & 5 & -4.8 & 2.4 & -4.3 & 2.6 \\
\hline $26 \mathrm{R}-1,28-30$ & 306.48 & 141 & Phyric massive basalt & WWU & $\mathrm{TH}$ & $360-520$ & 6 & -13.2 & 3.0 & -13.1 & 3.4 \\
\hline $26 \mathrm{R}-1,78-80$ & 306.98 & 142 & Phyric massive basalt & Shipboard & $\mathrm{AF}$ & $10-80$ & 10 & -3.7 & 1.2 & & \\
\hline $26 \mathrm{R}-2,40-42$ & 308.07 & 145 & Phyric massive basalt & WWU & $\mathrm{AF}$ & $20-45$ & 6 & 0.0 & 2.2 & -0.2 & 2.3 \\
\hline $26 \mathrm{R}-4,27-29$ & 310.54 & 156 & Phyric massive basalt & Shipboard & $\mathrm{TH}$ & $150-550$ & 14 & 4.7 & 1.9 & & \\
\hline $26 \mathrm{R}-6,12-14$ & 313.11 & 170 & Phyric massive basalt & WWU & $\mathrm{AF}$ & $25-45$ & 5 & -1.5 & 1.4 & -2.2 & 0.8 \\
\hline 26R-7, 13-15 & 314.35 & 176 & Phyric massive basalt & WWU & $\mathrm{AF}$ & $10-45$ & 8 & -0.8 & 1.2 & -0.8 & 1.3 \\
\hline $26 \mathrm{R}-8,11-13$ & 315.40 & 181 & Phyric massive basalt & Shipboard & $\mathrm{TH}$ & $150-550$ & 14 & 4.2 & 3.3 & & \\
\hline $26 \mathrm{R}-8,43-45$ & 315.72 & 183 & Phyric massive basalt & WWU & $\mathrm{AF}$ & $15-45$ & 7 & -2.2 & 0.7 & -2.2 & 0.7 \\
\hline
\end{tabular}

$\mathrm{AF}=$ alternating field, $\mathrm{TH}=$ thermal demagnetization, $\mathrm{Steps}=$ demagnetization steps used to calculate principal component direction, $N=$ number of points in used in principle component calculation, Inc = inclination, MAD = maximum angular deviation, anc = principal component analysis calculated with anchor at origin. WWU $=$ Northwest Paleomagnetism Laboratory at Western Washington University (USA).

Table T4. Change in magnetic intensity for samples exposed to low temperature, Hole U1346A.

\begin{tabular}{cccc}
\hline $\begin{array}{c}\text { Core, section, } \\
\text { interval }(\mathrm{cm})\end{array}$ & $\begin{array}{c}\text { NRM } \\
(\mathrm{A} / \mathrm{m})\end{array}$ & $\begin{array}{c}\text { Magnetization } \\
\text { after final treatment } \\
(\mathrm{A} / \mathrm{m})\end{array}$ & $\begin{array}{c}\text { Difference } \\
(\mathrm{A} / \mathrm{m})\end{array}$ \\
\hline 324-U1346A- & & & \\
10R-1, 40 & 0.79 & 0.84 & -0.04 \\
13R-1, 5 & 5.02 & 4.48 & 0.55 \\
14R-2, 61 & 7.28 & 7.53 & -0.25 \\
8R-1, 136 & 2.20 & 2.23 & -0.03 \\
\hline
\end{tabular}

$\mathrm{NRM}=$ natural remanent magnetization 
Table T5. Change in magnetic intensity for samples exposed to low temperature, Hole U1347A.

\begin{tabular}{|c|c|c|c|}
\hline $\begin{array}{l}\text { Core, section, } \\
\text { interval }(\mathrm{cm})\end{array}$ & $\begin{array}{l}\text { NRM } \\
(A / m)\end{array}$ & $\begin{array}{c}\text { Magnetization } \\
\text { after final treatment } \\
(\mathrm{A} / \mathrm{m})\end{array}$ & $\begin{array}{l}\text { Difference } \\
(\mathrm{A} / \mathrm{m})\end{array}$ \\
\hline \multicolumn{4}{|l|}{ 324-U1347A- } \\
\hline $12 \mathrm{R}-1,104$ & 6.46 & 5.59 & 0.87 \\
\hline $12 \mathrm{R}-1,93$ & 10.65 & 7.83 & 2.81 \\
\hline 12R-2, 2 & 9.30 & 7.75 & 1.55 \\
\hline $12 \mathrm{R}-2,81$ & 7.43 & 6.58 & 0.84 \\
\hline $13 \mathrm{R}-1,7$ & 28.55 & 24.55 & 4.01 \\
\hline $13 \mathrm{R}-2,14$ & 18.11 & 15.44 & 2.67 \\
\hline $13 \mathrm{R}-3,23$ & 2.44 & 1.76 & 0.69 \\
\hline 13R-6, 132 & 6.66 & 5.68 & 0.98 \\
\hline 14R-1, 51 & 5.35 & 5.07 & 0.27 \\
\hline $14 \mathrm{R}-1,84$ & 4.71 & 4.07 & 0.64 \\
\hline $14 \mathrm{R}-2,110$ & 12.78 & 12.33 & 0.45 \\
\hline $15 \mathrm{R}-2,15$ & 9.02 & 8.91 & 0.11 \\
\hline 16R-1, 21 & 11.27 & 7.75 & 3.52 \\
\hline 16R-2, 14 & 2.90 & 3.20 & -0.30 \\
\hline 16R-3, 29 & 21.22 & 16.06 & 5.16 \\
\hline $17 \mathrm{R}-2,94$ & 8.49 & 7.97 & 0.52 \\
\hline $17 \mathrm{R}-3,86$ & 11.31 & 11.96 & -0.64 \\
\hline $18 \mathrm{R}-4,54$ & 14.41 & 14.55 & -0.14 \\
\hline $18 \mathrm{R}-5,66$ & 15.05 & 15.13 & -0.07 \\
\hline 19R-3, 14 & 11.05 & 10.44 & 0.61 \\
\hline $20 \mathrm{R}-3,5$ & 18.68 & 17.80 & 0.88 \\
\hline $22 \mathrm{R}-1,51$ & 6.17 & 6.18 & -0.01 \\
\hline $23 \mathrm{R}-3,62$ & 7.32 & 7.32 & -0.01 \\
\hline $24 \mathrm{R}-3,85$ & 10.50 & 10.70 & -0.20 \\
\hline $25 R-4,125$ & 5.93 & 5.87 & 0.06 \\
\hline $27 \mathrm{R}-1,74$ & 11.75 & 10.03 & 1.72 \\
\hline $27 \mathrm{R}-2,78$ & 30.21 & 27.10 & 3.11 \\
\hline $27 R-3,65$ & 36.50 & 31.22 & 5.28 \\
\hline $27 \mathrm{R}-3,8$ & 22.36 & 18.96 & 3.40 \\
\hline $27 \mathrm{R}-4,21$ & 42.71 & 37.46 & 5.28 \\
\hline $27 \mathrm{R}-5,120$ & 40.84 & 34.88 & 5.96 \\
\hline $27 \mathrm{R}-5,4$ & 16.10 & 13.57 & 2.53 \\
\hline $27 R-6,22$ & 43.14 & 38.72 & 4.42 \\
\hline $28 \mathrm{R}-1,8$ & 47.05 & 39.70 & 7.36 \\
\hline $28 \mathrm{R}-2,28$ & 29.97 & 21.34 & 8.63 \\
\hline $28 \mathrm{R}-7,13$ & 36.96 & 31.48 & 5.48 \\
\hline $29 \mathrm{R}-3,68$ & 40.63 & 30.70 & 9.94 \\
\hline $29 \mathrm{R}-4,106$ & 5.14 & 3.45 & 1.69 \\
\hline $29 \mathrm{R}-5,86$ & 21.93 & 20.90 & 1.04 \\
\hline
\end{tabular}

$\mathrm{NRM}=$ natural remanent magnetization. 
Table T6. Change in magnetic intensity for samples exposed to low temperature, Hole U1350A.

\begin{tabular}{|c|c|c|c|}
\hline $\begin{array}{l}\text { Core, section, } \\
\text { interval }(\mathrm{cm})\end{array}$ & $\begin{array}{l}\text { NRM } \\
(\mathrm{A} / \mathrm{m})\end{array}$ & $\begin{array}{c}\text { Magnetization } \\
\text { after final treatment } \\
(\mathrm{A} / \mathrm{m})\end{array}$ & $\begin{array}{c}\text { Difference } \\
(A / m)\end{array}$ \\
\hline \multicolumn{4}{|l|}{ 324-U1350A- } \\
\hline 10R-1, 21 & 2.41 & 1.55 & 0.86 \\
\hline 10R-2, 38 & 11.41 & 10.87 & 0.53 \\
\hline $11 \mathrm{R}-1,128$ & 11.57 & 11.25 & 0.32 \\
\hline $11 \mathrm{R}-2,14$ & 8.23 & 7.43 & 0.79 \\
\hline $12 \mathrm{R}-1,8$ & 12.94 & 11.94 & 1.00 \\
\hline $13 \mathrm{R}-1,100$ & 2.66 & 2.64 & 0.02 \\
\hline $13 R-2,55$ & 3.31 & 2.98 & 0.34 \\
\hline $14 \mathrm{R}-1,52$ & 9.38 & 9.08 & 0.30 \\
\hline 14R-2, 21 & 15.54 & 15.22 & 0.31 \\
\hline 15R-1, 101 & 4.16 & 2.48 & 1.68 \\
\hline $15 R-1,53$ & 10.92 & 10.81 & 0.11 \\
\hline 16R-2, 5 & 4.97 & 4.88 & 0.09 \\
\hline 16R-3, 105 & 28.40 & 27.64 & 0.76 \\
\hline $17 \mathrm{R}-1,138$ & 7.76 & 7.90 & -0.15 \\
\hline $17 \mathrm{R}-2,80$ & 7.34 & 7.19 & 0.14 \\
\hline $18 \mathrm{R}-1,72$ & 7.23 & 7.09 & 0.15 \\
\hline $18 \mathrm{R}-2,73$ & 9.62 & 9.12 & 0.50 \\
\hline 18R-3, 12 & 11.57 & 10.93 & 0.63 \\
\hline $19 \mathrm{R}-3,37$ & 7.65 & 7.54 & 0.11 \\
\hline $25 \mathrm{R}-2,60$ & 13.68 & 14.35 & -0.67 \\
\hline $25 \mathrm{R}-8,13$ & 8.88 & 9.26 & -0.38 \\
\hline $26 \mathrm{R}-7,13$ & 5.78 & 5.81 & -0.03 \\
\hline 7R-1, 104 & 8.25 & 7.99 & 0.26 \\
\hline 7R-2, 21 & 6.88 & 4.71 & 2.17 \\
\hline $8 R-1,63$ & 5.38 & 4.71 & 0.67 \\
\hline $8 \mathrm{R}-2,4$ & 18.06 & 17.79 & 0.27 \\
\hline $8 \mathrm{R}-2,114$ & 5.54 & 4.76 & 0.78 \\
\hline $8 R-3,4$ & 6.41 & 6.37 & 0.04 \\
\hline $9 R-1,58$ & 4.34 & 3.82 & 0.52 \\
\hline $9 \mathrm{R}-3,51$ & 3.44 & 3.27 & 0.17 \\
\hline 9R-5, 9 & 1.79 & 1.55 & 0.24 \\
\hline $9 R-6,67$ & 2.24 & 1.64 & 0.59 \\
\hline
\end{tabular}

$\mathrm{NRM}=$ natural remanent magnetization.

Table T7. Sample hysteresis parameters, Holes U1346A, U1347A, and U1350A.

\begin{tabular}{ccccrrr}
\hline $\begin{array}{c}\text { Core, section, } \\
\text { interval (cm) }\end{array}$ & $M_{\mathrm{rs}}$ & $M_{\mathrm{s}}$ & $M_{\mathrm{rs}} / M_{\mathrm{s}}$ & $H_{\mathrm{cr}}$ & $H_{\mathrm{c}}$ & $H_{\mathrm{cl}} / H_{\mathrm{c}}$ \\
\hline 324-U1346A- & & & & & & \\
$10-1,40$ & 0.000223 & 0.001040 & 0.214327 & 99.1 & 73.6 & 1.3 \\
$10-2,75$ & 0.000048 & 0.000794 & 0.060645 & 62.9 & 21.0 & 3.0 \\
$13-2,59$ & 0.000160 & 0.000870 & 0.182874 & 178.2 & 103.3 & 1.7 \\
$14-1,136$ & 0.000133 & 0.001028 & 0.129280 & 121.3 & 53.4 & 2.3 \\
$22-2,74$ & 0.001471 & 0.007274 & 0.202227 & 114.4 & 77.2 & 1.5 \\
$324-\mathrm{U} 1347 \mathrm{~A}-$ & & & & & & \\
$22-5,61$ & 0.001212 & 0.003515 & 0.344808 & 401.0 & 293.4 & 1.4 \\
$24-8,65$ & 0.002789 & 0.007543 & 0.369747 & 416.0 & 381.2 & 1.1 \\
$24-8,122$ & 0.000250 & 0.001464 & 0.171038 & 116.9 & 72.9 & 1.6 \\
$324-\mathrm{U} 1350 \mathrm{~A}-$ & & & & & & \\
$22-5,135$ & 0.001937 & 0.005828 & 0.332361 & 393.2 & 272.7 & 1.4 \\
$23-2,58$ & 0.000164 & 0.001013 & 0.162290 & 101.2 & 63.0 & 1.6 \\
$25-1,78$ & 0.000398 & 0.001855 & 0.214663 & 130.7 & 91.7 & 1.4 \\
$25-1,112$ & 0.000640 & 0.002915 & 0.219383 & 284.1 & 146.5 & 1.9 \\
\hline
\end{tabular}

$M_{\mathrm{rs}}=$ saturation remanent magnetization, $M_{\mathrm{s}}=$ saturation magnetization, $H_{\mathrm{cr}}=$ remanent coercivity, $H_{\mathrm{c}}=$ coercivity . 